Check for updates

Cite this: RSC Adv., 2018, 8, 1296

Received 3rd October 2017

Accepted 14th December 2017

DOI: $10.1039 / c 7 r a 10929 f$

rsc.li/rsc-advances

\section{Silylated oligomeric poly(ether-azomethine)s from monomers containing biphenyl moieties: synthesis and characterization}

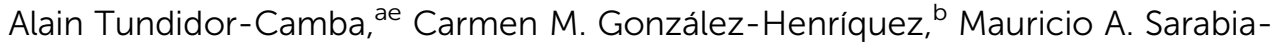 \\ Vallejos, ${ }^{c}$ Luis H. Tagle, ${ }^{a}$ René A. Hauyón, ${ }^{a}$ Patricio A. Sobarzo, ${ }^{a}$ Alexis González, ${ }^{a}$ \\ Pablo A. Ortiz, ${ }^{a}$ Eva M. Maya (D) and Claudio A. Terraza (D) *ae
}

\begin{abstract}
In this study, four new silicon-containing poly(ether-azomethine)s with linear structures were prepared using original silicon and biphenyl moiety-containing monomers: two diamines and two dialdehydes. The oligomeric natures of the samples were established by GPC analysis, which showed chains containing 3 to 5 repetitive units. The monomers and the oligomeric samples were structurally characterized by NMR and FT-IR spectroscopy. The solubilities of the samples in common organic solvents and their thermal behavior enable improvement of their industrial and technological processability. The optical band gaps of the oligomeric samples were estimated from optical measurements (UV-vis), and their electrical behavior in films was determined using the four-point method. The surface arrangements and morphological characteristics of the films were determined via atomic force microscopy measurements. The roughness, area increase percentage and layer stiffness of the films were also measured using this technique.
\end{abstract}

\section{Introduction}

Conjugated polymers have recently been used as photovoltaic compounds in organic bulk heterojunctions due to their intrinsic properties, such as their small bandgaps and good hole transportation. Their ease of processing and low cost in comparison to inorganic semiconductors, namely silicon, make them excellent alternatives for this purpose. ${ }^{1}$ On this basis, the synthesis of organometallic polymers with silicon atoms in their structures may unify the features of a suitable band gap with electron delocalization, high voltage, and low sub-bandgap absorption, among other properties.

Conjugated poly(azomethine)s (PAMs) are a family of polymers which possess an imine repeating unit, $\mathrm{R}-\mathrm{HC}=\mathrm{N}-\mathrm{R}^{\prime}$, where $\mathrm{R}$ and $\mathrm{R}^{\prime}$ are moieties that allow conjugation (e.g. aromatic moieties). ${ }^{2}$ These materials have been the subject of extensive investigation, especially in the last three decades; due

${ }^{a}$ Research Laboratory for Organic Polymers (RLOP), Department of Organic Chemistry, Pontificia Universidad Católica de Chile, P.O. Box. 306, Post 22, Santiago, Chile. E-mail: cterraza@uc.cl

${ }^{b}$ Laboratory of Nanotechnology and Advanced Materials (LNNMA), Chemistry Department, Universidad Tecnológica Metropolitana, P.O. Box 9845, Post 21, Santiago, Chile

'Departamento de Ingeniería Estructural y Geotecnia, Escuela de Ingeniería, Pontificia Universidad Católica de Chile, Santiago, Chile

${ }^{d}$ Instituto de Ciencia de Materiales de Madrid, Consejo Superior de Investigaciones Cientificas, CSIC, Spain

${ }^{e}$ UC Energy Research Center, Pontifica Universidad Católica de Chile, Chile to their optoelectronic, non-linear optic and electroluminescent behaviors, they are excellent candidates as materials to be used in the fabrication of organic light-emitting diodes (OLEDs), photovoltaic cells, and $\mathrm{pH}$ sensors, among other applications. ${ }^{3}$

Early studies on the synthesis of PAMs led to low molecular weight polymers and intractable materials (both infusible and insoluble); these characteristics hampered their potential applications due to their low processability. ${ }^{4}$ This, in turn, excluded the possibility of fabricating films, thus limiting the characterization of these materials apart from hightemperature processability leading to the fabrication of fibers. ${ }^{5}$ The ability to form films is desirable today for the fabrication of devices such as OLEDs. ${ }^{6}$

In recent years, many approaches have been used to solve this problem: namely, the introduction of side groups in the polymer chain ${ }^{7}$ and their complexation with Brønsted acids such as diphenyl-phosphate and di-m-cresyl phosphate and Lewis acids such as gallium chloride to interact with the nitrogen atom of the imine functional group. ${ }^{5}$ These strategies enhanced the solubility of PAMs in common organic solvents and affected the thermal behavior of the polymers, achieving meltability without compromising the high thermal stability that characterizes this family of materials.

Another widely used strategy to achieve better processability of polymers is the introduction of flexible $-\operatorname{Ar}-\operatorname{Si}\left(R_{1} R_{2}\right)-\operatorname{Ar}-$ units, in which the $\mathrm{R}_{1}$ and $\mathrm{R}_{2}$ groups are alkyl or phenyl units. In this sense, this strategy has already proved useful in the preparation of highly thermally stable poly(ester)s and poly(amide)s 
with glass transition temperatures $\left(T_{\mathrm{g}}\right)$ below their thermal decomposition temperatures (TDT) and with high solubility in common organic solvents. ${ }^{8}$

The - $\mathrm{Ar}-\mathrm{Si}\left(\mathrm{R}_{1} \mathrm{R}_{2}\right)-\mathrm{Ar}-$ moiety has not only been investigated in the improvement of the processability of polymers. More recently, this unit has been used in the creation of materials for phosphorescent organic light-emitting diodes (PHOLEDs), ${ }^{9-13}$ separation and capture of $\mathrm{CO}_{2}$ gas, ${ }^{14,15}$ membrane fabrication for gas separation applications ${ }^{16}$ fabrication of low-cost polymer solar cells ${ }^{17,18}$ and synthesis of porous aromatic frameworks. ${ }^{19}$

In the case of PAMs, Iwan and $\mathrm{Sek}^{3}$ have provided an extensive review that considers the applications of these polymers, ranging from the aerospace industry to catalyst carriers, ion complexing agents, and anticorrosion materials as well as in the fabrication of photovoltaic cells and OLEDs.

Considering the interesting properties of both the $-\mathrm{Ar}-$ $\mathrm{Si}\left(\mathrm{R}_{1} \mathrm{R}_{2}\right)-\mathrm{Ar}-$ unit and the PAMs family, it is quite worthwhile to investigate their use in the same macromolecular material. The scientific literature provides very few studies devoted to this subject. In fact, in studies in which the silicon atom is incorporated into the poly(azomethine) backbone, it is not part of a $\mathrm{Ar}-\mathrm{Si}\left(\mathrm{R}_{1} \mathrm{R}_{2}\right)-\mathrm{Ar}-$ unit. In general, the silicon atom is part of a siloxane moiety, which implies a Si-O bond. ${ }^{20-25}$ In 2014, Zaltariov et al. ${ }^{26}$ reported a PAM with a central silicon atom bonded to four carbon atoms complexed with different metal ions. However, they used a methylene unit instead of an aromatic moiety bonded to a silicon atom which, along with other structural features, rendered the polymer too flexible; thus, the $T_{\mathrm{g}}$ measured for the polymer was $48^{\circ} \mathrm{C}$. For a material to be used for charge transport in a PHOLED device, its $T_{\mathrm{g}}$ value must be over $100{ }^{\circ} \mathrm{C}$ to avoid deterioration of its mechanical properties under high temperature conditions. ${ }^{9}$

The aim of this work is the synthesis and chemical and structural characterization of four oligomeric poly(etherazomethine)s, designated PAM-I, PAM-II, PAM-III and PAM-IV, which contain silicon atoms and imino functions in their main chains in addition to methyl or phenyl groups in their lateral segments. Thus, the structural properties of the materials were studied using nuclear magnetic resonance (NMR) spectroscopy, Fourier transform infrared (FT-IR) spectroscopy and elemental analyses. The optical properties of the samples were measured via UV-vis spectroscopy (optical absorption). Their solubilities were tested in several organic solvents, and their thermal behavior was established by thermogravimetric analysis (TGA) and differential scanning calorimetry (DSC). Finally, two different films were prepared using the spin-coating technique for deposition, using silicon wafers previously treated with piranha solution $\left(\mathrm{H}_{2} \mathrm{SO}_{4}: \mathrm{H}_{2} \mathrm{O}_{2}\right)$ as the substrate. The samples selected were PAM-I and PAM-II; both dissolved in DMAc. The thicknesses of the films were determined by ellipsometry using the refractive index of each layer, determined through an Abbe refractometer. Morphological representations of the film surfaces were obtained by atomic force microscopy (AFM). Additionally, conductivity values for the solid films were also determined through the four-point method, using a silicon wafer as the calibration material.

\section{Experimental section}

\section{Materials}

$n$-Butyllithium solution ( $2 \mathrm{M}$ in cyclohexane), dimethyldichlorosilane, diphenyldichlorosilane, bis(triphenylphosphine) palladium(II) dichloride, anhydrous $N, N$-dimethylacetamide (DMAc), anhydrous calcium chloride, $p$-toluenesulfonic acid, 1fluoro-4-nitrobenzene, hydrazine monohydrate (80\%) and $\mathrm{Pd} / \mathrm{C}$ $(10 \% \mathrm{w} / \mathrm{w})$ were obtained from Aldrich Chemical (Milwaukee, WI, USA). 4-Hydroxyphenylboronic acid and 4-formylphenylboronic acid were purchased from AK Scientific, Inc. (San Francisco, USA). All other reagents and solvents were purchased commercially as analytical grade (Aldrich Chemical, WI, USA or Merck, Darmstadt, Germany). Immediately before use, diethyl ether was refluxed with sodium under nitrogen atmosphere and then distilled.

\section{Measurements}

NMR spectra were acquired on a $400 \mathrm{MHz}$ instrument (Bruker AC-200, Germany) using $\mathrm{CDCl}_{3}$ or DMSO- $d_{6}$ as solvent for the precursors and monomers, while DMAc-acetone- $d_{6}$ was employed for the oligomeric samples. In all cases, TMS was added as an internal standard. FT-IR spectra ( $\mathrm{KBr}$ pellets) were recorded on a Perkin-Elmer (Fremont CA, USA) 1310 spectrophotometer over the range of 4000 to $450 \mathrm{~cm}^{-1}$. The melting points of the precursors and monomers were obtained using a Stuart Scientific SMP3 instrument. Elemental analyses were conducted on Fisons EA 1108-CHNS-O equipment (Thermo Scientific, Waltham/MA, USA). Viscosimetric measurements were performed in a Desreux-Bischof-type dilution viscosimeter at $25{ }^{\circ} \mathrm{C}\left(c=0.5 \mathrm{~g} \mathrm{dL}^{-1}\right)$. The weight-average $\left(M_{\mathrm{w}}\right)$ and numberaverage $\left(M_{\mathrm{n}}\right)$ molecular weights, degrees of polymerization (DP) and polydispersity indices (PDI) of the oligomeric samples relative to polyethylene glycol oxide standards were determined at $40{ }^{\circ} \mathrm{C}$ using a GPC $150 \mathrm{cv}$ system (Waters, USA) equipped with a refractive index detector. For this, solutions of oligomeric samples in DMF-0.05 M LiBr $\left(c=0.5 \mathrm{mg} \mathrm{mL}^{-1}\right)$ were filtered through micro-filters with pores of $2 \mu \mathrm{m}$; then, $100 \mu \mathrm{L}$ of sample was injected at $1 \mathrm{~mL} \mathrm{m^{-1 }}$ in a MesoPore column $(300 \times 75$ $\mathrm{mm}$ ), which is suitable for samples with molecular weights up to 25 000. Glass transition temperatures were obtained with a Mettler-Toledo (Greifensee, Switzerland) DSC 821 calorimetric system $\left(20{ }^{\circ} \mathrm{C} \min ^{-1}\right.$ under $\mathrm{N}_{2}$ flow) after the second heating scan. Thermogravimetric analyses were carried out on a Mettler (Switzerland) TA-3000 calorimetric system equipped with a TC10A processor and a TG-50 thermobalance with a Mettler MT5 microbalance. Samples of 6 to $10 \mathrm{mg}$ were placed in an alumina sample holder, and the thermogravimetric measurements were carried out between $25{ }^{\circ} \mathrm{C}$ and $800{ }^{\circ} \mathrm{C}$ with a heating rate of $20{ }^{\circ} \mathrm{C} \min ^{-1}$ under $\mathrm{N}_{2}$ flow. The absorption spectra of the polymers were recorded at room temperature between 270 and $440 \mathrm{~nm}$ using a Lambda 35 model UV-vis spectrophotometer (Perkin Elmer, USA) using solutions of DMSO $\left(c=0.5 \mathrm{~g} \mathrm{~L}^{-1}\right)$.

A spin coater, model KW-4A from Chemat Scientific, coupled with an oil-free vacuum pump (Rocker Chemker 410) was used for deposition of the polymer dissolved in DMAc over the 
pretreated silicon wafer. This instrument possesses two spinning stages: (1) 500 to $2500 \mathrm{rpm}$ (2 to 18 seconds) and (2) 800 to $8000 \mathrm{rpm}$ (3 to 60 seconds) with a tunable acceleration rate. A Bresser Trino Researcher II (40-1000X) trinocular microscope coupled with a CCD color camera (5 Mp, Bresser) and a cold light source (Optika CL-41) was used as the first approach for visualizing the topographies of the polymeric films. The surface topographies of the polymers were obtained at room temperature using an NTEGRA Prima AFM (NT-MDT Co.) in intermittent contact mode at different scan ranges $\left(25 \times 25 \mu \mathrm{m}^{2}\right.$ and 50 $\times 50 \mu^{2}$ ). Force spectroscopy measurements were performed with a NaioAFM atomic force microscope (Nanosurf Inc.) using a conical contact mode tip. These results were analyzed through the model proposed by Hertz for tip indentation over soft surfaces according to Roa et $a .^{27}$ Images were treated and analyzed using the off-line software Gwyddion. ${ }^{28}$ A multi-angle laser ellipsometer, model SE 400adv, from SENTECH Instruments $\mathrm{GmbH}$ was used to perform optical measurements with variable incidence angles from $30^{\circ}$ to $90^{\circ}$ in steps of $0.5^{\circ}$; the equipment possessed an attached motorized goniometer (Hüber Diffraktionstechnik GmbH \& Co. KG) for control incidence angle variation. A stabilized He-Ne laser $(\lambda=633 \mathrm{~nm})$ permitted a precision of $0.1 \AA$ in the thin film thickness measurements. This equipment was used to measure the thicknesses of the polymeric films (PAM-I and PAM-II). Refractive indices of these polymers were obtained using an AbbeRefractometer model AR4 from A. KRÜSS Optronic GmbH in DMAc solution at room temperature. The four-point method was carried out using a digital multimeter from GW Instek, model GDM-8255, which includes a $4 \mathrm{~W}$ test lead for conduction measurements as an optional accessory. A DC power supply from GW Instek, model PLR 20-18, was used to administer a constant current to the system $(6 \mathrm{~A})$.

\section{Polymeric film preparation}

Silicon wafer cleaning method. The surface nature of the silicon wafer $(\mathrm{Si}(100))$ was modified through a specific treatment which improves the hydrophilicity of the surface. The substrate was first immersed in a solution for increased surface density, leaving hydroxylated oxide at the top level. This pretreatment involves a piranha solution that removes the original native silicon oxide film. The silicon wafer was placed in the piranha solution $\left(\mathrm{H}_{2} \mathrm{SO}_{4}: \mathrm{H}_{2} \mathrm{O}_{2} ; 7: 3 \mathrm{vol} / \mathrm{vol}\right)$ for 30 minutes at $80{ }^{\circ} \mathrm{C}^{29,30}$ Afterward, the wafers were washed with HPLC water and slightly sonicated in order to eliminate traces of sulfuric acid. Then, the wafers were dried with an ultra-pure nitrogen gas jet. This substrate treatment is important to improve the adherence of the polymer when the film is formed.

Thin film deposition using spin coating. $9.5 \mathrm{mg}$ of each sample were dissolved in $500 \mu \mathrm{L}$ of DMAc. Then, $20 \mu \mathrm{L}$ of each dissolved sample were deposited over a previously treated hydrophilic silicon wafer $\left(1 \times 1 \mathrm{~cm}^{2}\right)$. The deposition parameters for the same compound were progressively varied by changing the acceleration rate (rpm) of the spin deposition. Afterward, the films were subjected to strong vacuum $\left(10^{-4}\right.$ torr $)$ for a week.

\section{Precursors and monomer synthesis}

Bis(4-bromophenyl)di-R-silane ( $R=$ methyl (1), phenyl (2)). Under nitrogen atmosphere, $n$-BuLi in cyclohexane $(2 \mathrm{M})$ (42.4 mL, $84.78 \mathrm{mmol}$ ) was slowly added to a solution of 1,4dibromobenzene $(20.00 \mathrm{~g}, 84.78 \mathrm{mmol})$ in $300 \mathrm{~mL}$ of anh. $\mathrm{Et}_{2} \mathrm{O}$ at $0{ }^{\circ} \mathrm{C}$. After stirring for one hour, a solution of dichlorodi-R-silane $(42.39 \mathrm{mmol})$ in $20 \mathrm{~mL}$ of anh. $\mathrm{Et}_{2} \mathrm{O}$ was added, and the mixture was stirred for 12 hours. The obtained white solid was filtered, and the solution was washed with water. The organic phase was dried with anh. $\mathrm{Na}_{2} \mathrm{SO}_{4}$ and concentrated to obtain a yellow oil. Cold methanol was used to obtain the dibromo derivatives as white solids; these were filtered, washed with cold methanol and dried at $40{ }^{\circ} \mathrm{C}$ under vacuum ( $\mathrm{R}=$ methyl, $79 \%$ and $\mathrm{R}=$ phenyl, 71\%). The products were recrystallized from acetone with yields close to $80 \%$.
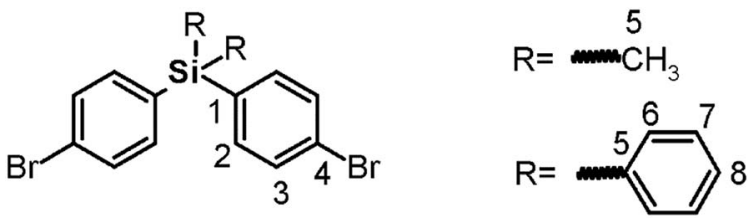

Bis(4-bromophenyl)dimethylsilane (1). $\mathrm{Mp}=73{ }^{\circ} \mathrm{C}$ to $74{ }^{\circ} \mathrm{C}$. IR-TF (KBr, $\nu, \mathrm{cm}^{-1}$ ): 3067, 3046, 3031, 3011 (C-H arom.); 2960 (C-H aliph.); 1566 (C=C); 1373 (Si-Ph); $1253\left(\mathrm{Si}-\mathrm{CH}_{3}\right) ; 832$ (arom. $p$-subst.); 500 (C-Br). ${ }^{1} \mathrm{H}$ NMR $\left(\mathrm{CDCl}_{3}, \delta, \mathrm{ppm}\right): 0.44$ (s, $6 \mathrm{H}, \mathrm{H} 5) ; 7.27$ (d, $J=7.91 \mathrm{~Hz}, 4 \mathrm{H}, \mathrm{H} 2) ; 7.39$ (d, $J=7.86 \mathrm{~Hz}, 4 \mathrm{H}$, $\mathrm{H} 3) .{ }^{13} \mathrm{C} \mathrm{NMR}\left(\mathrm{CDCl}_{3}, \delta, \mathrm{ppm}\right):-2.42$ (C5); 124.38 (C4); 131.23 (C3), 135.82 (C2), 136.55 (C1). ${ }^{29} \mathrm{Si} \mathrm{NMR}\left(\mathrm{CDCl}_{3}, \delta\right.$, ppm): -7.00. Elem. anal. calcd for $\mathrm{C}_{14} \mathrm{H}_{14} \mathrm{Br}_{2} \mathrm{Si}$; (370.17): C, $45.42 \%$; $\mathrm{H}, 3.82 \%$. Found: C, 45.39\%; H, 3.79\%.

Bis(4-bromophenyl)diphenylsilane (2). $\mathrm{Mp}=159{ }^{\circ} \mathrm{C}$ to $164{ }^{\circ} \mathrm{C}$. FT-IR (KBr, $\nu, \mathrm{cm}^{-1}$ ): 3063, 3023 (C-H arom.); 1568 (C= C); 1427, 729 (Si-Ph); 809 (arom. $p$-subst); 512 (C-Br). ${ }^{1} \mathrm{H}$ NMR $\left(\mathrm{CDCl}_{3}, \delta, \mathrm{ppm}\right): 7.37$ (m, 4H, H7); 7.39 (m, 4H, H2); 7.44 (m, 2H, $\mathrm{H} 8) ; 7.51$ (m, 8H, H3, H6). ${ }^{13} \mathrm{C} \mathrm{NMR}\left(\mathrm{CDCl}_{3}, \delta, \mathrm{ppm}\right): 123.94$ (C4); 127.07 (C7); 128.97 (C8); 130.18 (C3), 131.62 (C1), 131.97 (C5), 135.18 (C6), 136.78 (C2). ${ }^{29} \mathrm{Si} \mathrm{NMR}\left(\mathrm{CDCl}_{3}, \delta, \mathrm{ppm}\right)$ : -14.01. Elem. anal. calcd for $\mathrm{C}_{24} \mathrm{H}_{18} \mathrm{Br}_{2} \mathrm{Si}$; (494.31): C, 58.31\%; H, 3.68\%. Found: C, 58.26\%; H, 3.61\%.

Bis(4-(4-hydroxyphenyl)phenyl)di-R-silane ( $R=$ methyl (3), phenyl (4)). To a mixture of dibromo derivative (1 or 2) (13.51 mmol), 4-hydroxyphenylboronic acid (4.66 g, $33.78 \mathrm{mmol}$ ) and bis(triphenylphosphine) palladium(II) dichloride (0.40 g, 0.570 $\mathrm{mmol})$, a solution containing $\mathrm{K}_{2} \mathrm{CO}_{3}(12.44 \mathrm{~g})$, water $(45 \mathrm{~mL})$ and 1,4-dioxane (45 mL) was added; then, the mixture was heated at $85{ }^{\circ} \mathrm{C}$ for 12 hours. After adding $100 \mathrm{~mL}$ of water, a brown solid was obtained for $\mathrm{R}=$ methyl and a black/brownoil was obtained for $\mathrm{R}=$ phenyl. The solids were recovered through filtration and treated with acetone and chloroform, respectively; then, the samples were filtered through celite. After drying and filtering, the solutions were concentrated to produce a brown solid for derivative 3 (94\%), which was recrystallized from abs. ethanol, and a light brown oil for 4. This oil was dissolved in chloroform and then added to $n$-hexane to obtain 
a grayish-white solid which was filtered, washed with $n$-hexane and dried at $60{ }^{\circ} \mathrm{C}$ under vacuum $(88 \%)$.
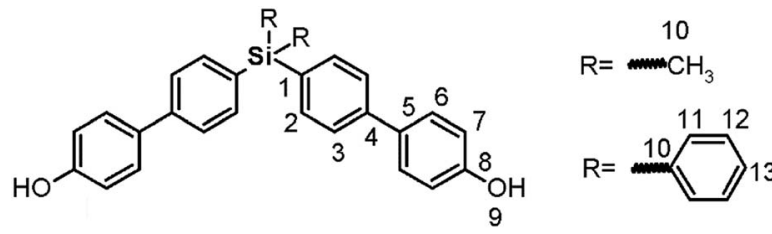

Bis(4-(4-hydroxyphenyl)phenyl)dimethylsilane (3). $\mathrm{Mp}=$ $87{ }^{\circ} \mathrm{C}$ to $94{ }^{\circ} \mathrm{C}$. FT-IR $\left(\mathrm{KBr}, \nu, \mathrm{cm}^{-1}\right): 3493(\mathrm{O}-\mathrm{H})$; 3052, $3014(\mathrm{C}-$ $\mathrm{H}$ arom.); 2958 (C-H aliph.); 1520 (C=C); 1434 (Si-Ph); 1247 (Si$\mathrm{CH}_{3}$ ); 819 (arom. $p$-subst); ${ }^{1} \mathrm{H}$ NMR (DMSO- $\left.d_{6}, \delta, \mathrm{ppm}\right): 0.56$ (s, $6 \mathrm{H}, \mathrm{H} 10) ; 6.88$ (d, $J=8.54 \mathrm{~Hz}, 4 \mathrm{H}, \mathrm{H} 7) ; 7.50$ (d, $J=8.53 \mathrm{~Hz}, 4 \mathrm{H}$, H6); 7.55 (d, $J=8.15 \mathrm{~Hz}, 4 \mathrm{H}, \mathrm{H} 2), 7.58$ (d, $J=8.19 \mathrm{~Hz}, 4 \mathrm{H}, \mathrm{H} 3$ ), 9.59 (s, 2H, H9). ${ }^{13} \mathrm{C}$ NMR (DMSO- $\left.d_{6}, \delta, \mathrm{ppm}\right):-2.57$ (C10); 115.78 (C7); 125.45 (C3); 127.79 (C6); 130.73 (C5); 134.43 (C2); 135.44 (C1); 140.96 (C4); 157.34 (C8). ${ }^{29} \mathrm{Si}$ NMR (DMSO- $d_{6}, \delta$, ppm): -8.64. Elem. anal. calcd for $\mathrm{C}_{26} \mathrm{H}_{24} \mathrm{O}_{2} \mathrm{Si}$; (396.59): C, $78.74 \%$; H, 6.11\%. Found: C, 78.64\%; H, 6.08\%.

Bis(4-(4-hydroxyphenyl)phenyl)diphenylsilane (4). $\mathrm{Mp}=$ $159{ }^{\circ} \mathrm{C}$ to $164{ }^{\circ} \mathrm{C}$. FT-IR $\left(\mathrm{KBr}, \nu, \mathrm{cm}^{-1}\right): 3355(\mathrm{O}-\mathrm{H})$; 3066, 3045 , 3017 (C-H arom.); 1519 (C=C); 1427 (Si-Ph); 815 (arom. $p$ subst.). ${ }^{1} \mathrm{H}$ NMR: $\left(\mathrm{CDCl}_{3}, \delta\right.$, ppm): 6.89 (d, $\left.J=8.06 \mathrm{~Hz}, 4 \mathrm{H}, \mathrm{H} 7\right)$; 7.38 (d, $J=6.68 \mathrm{~Hz}, 4 \mathrm{H}, \mathrm{H} 12) ; 7.42$ (d, $J=6.97 \mathrm{~Hz}, 2 \mathrm{H}, \mathrm{H} 13)$; 7.47 (d, $J=8.22 \mathrm{~Hz}, 4 \mathrm{H}, \mathrm{H} 6) ; 7.54$ (d, $J=7.65 \mathrm{~Hz}, 4 \mathrm{H}, \mathrm{H} 3$ ); 7.62 (d, $J=7.03 \mathrm{~Hz}, 8 \mathrm{H}, \mathrm{H} 2$ y H11). 5.11 (s, 2H, H9). ${ }^{13} \mathrm{C} \mathrm{NMR}\left(\mathrm{CDCl}_{3}\right.$, $\delta, \mathrm{ppm}$ ): 115.77 (C7); 126.10 (C3); 127.92 (C12); 128.38 (C6); 129.62 (C13); 132.21 (C1); 133.48 (C5); 134.35 (C10); 136.42 and 136.87 (C2 and C11); 141.78 (C4), 155.41 (C8). ${ }^{29} \mathrm{Si} \mathrm{NMR}\left(\mathrm{CDCl}_{3}\right.$, $\delta$, ppm): -14.46. Elem. anal. calcd for $\mathrm{C}_{36} \mathrm{H}_{28} \mathrm{O}_{2} \mathrm{Si}$; (520.73): C, $83.03 \%$; H, 5.43\%. Found: C, 82.98\%; H, 5.37\%.

Bis(4-(4-(4-nitrophenoxy)phenyl)phenyl)di-R-silane $\quad(\mathrm{R}=$ methyl (5), phenyl (6)). A mixture of diphenol derivative (3 or 4) (11.90 $\mathrm{mmol})$, potassium carbonate $(3.37 \mathrm{~g}, 24.40 \mathrm{mmol})$ and $15 \mathrm{~mL}$ of DMF was stirred and heated at $60{ }^{\circ} \mathrm{C}$. To this mixture, 1-fluoro-4-nitrobenzene (3.36 g, $23.80 \mathrm{mmol})$ was added; the mixture was stirred for 12 hours while maintaining the temperature. The obtained solution was added to a waterethanol solution (1:1 vol/vol), and an ochre solid was formed. The product was filtered and washed with water and then purified through silica column chromatography. For isolation of 3 , an $n$-hexane-chloroform mixture (1:1 vol/vol)) was employed as the mobile phase. The residue obtained after solvent evaporation was recrystallized from ethyl acetate to afford a light yellow solid (88\%). For isolation of 4 , a petroleum ether-chloroform mixture $(1: 1 \mathrm{vol} / \mathrm{vol})$ was used, and the obtained residue was treated with diethyl ether to produce a light yellow solid (74\%).
Bis(4-(4-(4-nitrophenoxy)phenyl)phenyl)dimethylsilane (5). $\mathrm{Mp}=98^{\circ} \mathrm{C}$ to $103{ }^{\circ} \mathrm{C}$. FT-IR (KBr, $\left.\nu, \mathrm{cm}^{-1}\right): 3080,3067,3011$ (C$\mathrm{H}$ arom.); 2954 (C-H aliph.); 1584, 1486 (C=C); 1518, 1345 $\left(\mathrm{NO}_{2}\right) ; 1246$ (C-O-C); $1110(\mathrm{Si}-\mathrm{Ph}) ; 847\left(\mathrm{Si}-\mathrm{CH}_{3}\right) ; 819$ (arom $p$ subst.). ${ }^{1} \mathrm{H}$ NMR $\left(\mathrm{CDCl}_{3}, \delta\right.$, ppm): 0.64 (s, 6H, H13); 7.07 (d, $J=$ 9.06, 4H, H10); 7.16 (d, $J=8.51,4 \mathrm{H}, \mathrm{H} 7) ; 7.60$ (d, $J=7.76,4 \mathrm{H}$, H3); 7.65 (m, 8H, H2, H6); 8.21 (d, $J=9.13,4 \mathrm{H}, \mathrm{H} 11) .{ }^{13} \mathrm{C}$ NMR $\left(\mathrm{CDCl}_{3}, \delta, \mathrm{ppm}\right):-2.33$ (C13); 117.27 (C10); 120.77 (C7); 125.98 (C11); 126.46 (C3); 128.96 (C6); 134.81 (C2); 137.22 (C1); 138.28 (C5); 140.77 (C4); 142.78 (C12); 154.35 (C8); 163.21 (C9). ${ }^{29} \mathrm{Si}$ NMR $\left(\mathrm{CDCl}_{3}, \delta, \mathrm{ppm}\right):-7.83$. Elem. anal. calcd for $\mathrm{C}_{38} \mathrm{H}_{30} \mathrm{~N}_{2} \mathrm{O}_{6} \mathrm{Si}$; (638.79): C, 71.44\%; H, 4.74\%; N, 4.39\%. Found: C, $71.40 \%$; H, $4.68 \%$, N, $4.32 \%$.

Bis(4-(4-(4-nitrophenoxy)phenyl)phenyl)diphenylsilane (6). $\mathrm{Mp}=196{ }^{\circ} \mathrm{C}$ to $197^{\circ} \mathrm{C}$. FT-IR $\left(\mathrm{KBr}, \nu, \mathrm{cm}^{-1}\right): 3068,3046,3016$ (C-H arom.); 1587, $1486(\mathrm{C}=\mathrm{C}) ; 1517,1343\left(\mathrm{NO}_{2}\right)$; 1246 (C-OC), 1111 (Si-Ph), 818 (arom. $p$-subst.). ${ }^{1} \mathrm{H} \mathrm{NMR}\left(\mathrm{CDCl}_{3}, \delta, \mathrm{ppm}\right)$ : 7.06 (d, $J=9.10 \mathrm{~Hz}, 4 \mathrm{H}, \mathrm{H} 10) ; 7.16$ (d, $J=8.43 \mathrm{~Hz}, 4 \mathrm{H}, \mathrm{H} 7) ; 7.41$ (t, $J=7.40 \mathrm{~Hz}, 4 \mathrm{H}, \mathrm{H} 15) ; 7.47$ (m, 2H, H16); 7.62 (d, $J=8.10 \mathrm{~Hz}$, $4 \mathrm{H}, \mathrm{H} 3$ ); 7.64 (d, $J=9.44 \mathrm{~Hz}, 4 \mathrm{H}, \mathrm{H} 14), 7.67$ (d, $J=9.04 \mathrm{~Hz}, 4 \mathrm{H}$, H6); 7.70 (d, $J=7.31 \mathrm{~Hz}, 4 \mathrm{H}, \mathrm{H} 2), 8.21$ (d, $J=9.12 \mathrm{~Hz}, 4 \mathrm{H}, \mathrm{H} 11)$. ${ }^{13} \mathrm{C}$ NMR ( $\left.\mathrm{CDCl}_{3}, \delta, \mathrm{ppm}\right): 117.29$ (C10); 120.79 (C7); 125.98 (C11); 126.47 (C3); 128.03 (C15); 128.98 (C6); 129.81 (C16); 133.27 (C1); 133.94 (C13); 136.39 (C14); 137.00 (C2); 138.10 (C5); 141.13 (C4); 142.79 (C12); 154.45 (C8); 163.17 (C9). ${ }^{29} \mathrm{Si}$ NMR $\left(\mathrm{CDCl}_{3}, \delta, \mathrm{ppm}\right):-14.37$. Elem. anal. calcd for $\mathrm{C}_{48} \mathrm{H}_{34} \mathrm{~N}_{2} \mathrm{O}_{6} \mathrm{Si}$; (762.93): C, 75.56\%; H, 4.50\%; N, 3.67\%. Found: C, 75.51\%; H, $4.44 \%, \mathrm{~N}, 3.60 \%$.

$\operatorname{Bis(4-(4-(4-aminophenoxy)phenyl)phenyl)di-R-silane~}(\mathrm{R}=$ methyl (7), phenyl (8)). A mixture of dinitro derivative (5 or 6 ) (6.341 mmol), Pd/C 10\% w/w (250 mg) and $40 \mathrm{~mL}$ of THF was heated under reflux. Then, $10 \mathrm{~mL}$ of hydrazine monohydrate (80\%) was slowly added, and the mixture was stirred for 12 hours. The remaining catalyst was removed by filtration through celite, the mixture was washed with THF, and the obtained solution was concentrated. For the purification of the samples: 7, obtained as a white solid, was treated with water; after filtration, the solid was washed again with water and dried at $100{ }^{\circ} \mathrm{C}$ under vacuum. The ochre solid was purified by column chromatography (silica/chloroform) and then recrystallized from acetonitrile to obtain a light orange solid (57\%). 8 was obtained as a yellow solid; it was washed with water, then filtered and dried at $70{ }^{\circ} \mathrm{C}$ under vacuum. The compound was purified by chromatography (silica/chloroform) (69\%).

Bis(4-(4-(4-aminophenoxy)phenyl)phenyl)dimethylsilane (7). $\mathrm{Mp}=95{ }^{\circ} \mathrm{C}$ to $99{ }^{\circ} \mathrm{C}$. FT-IR $\left(\mathrm{KBr}, \nu, \mathrm{cm}^{-1}\right): 3342,3448(\mathrm{~N}-\mathrm{H})$; 3011, 3038, 3065 (C-H arom.); 2957 (C-H aliph.); 1598, 1505, 1483 (C=C); 1239 (C-O-C); 1111 ( $\mathrm{Si}-\mathrm{Ph}) ; 879\left(\mathrm{Si}-\mathrm{CH}_{3}\right) ; 811$ (arom. $p$-subst.). ${ }^{1} \mathrm{H}$ NMR $\left(\mathrm{CDCl}_{3}, \delta, \mathrm{ppm}\right): 0.59$ (s, 6H, H13);

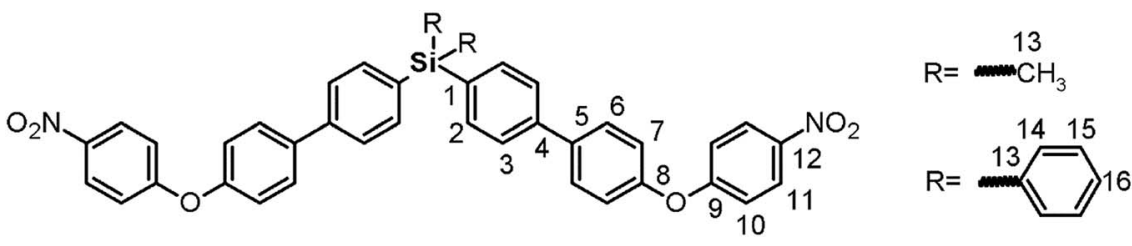




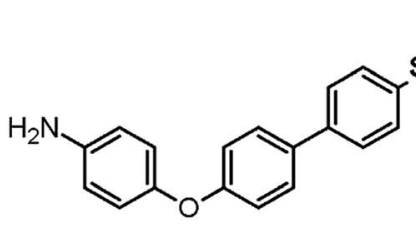

3.57 (s, 4H, H17); 6.68 (d, $J=8.33 \mathrm{~Hz}, 4 \mathrm{H}, \mathrm{H} 11) ; 6.90$ (d, $J=$ $7.14 \mathrm{~Hz}, 4 \mathrm{H}, \mathrm{H} 10) ; 6.98$ (d, $J=7.36 \mathrm{~Hz}, 4 \mathrm{H}, \mathrm{H} 7) ; 7.50$ (d, $J=$ $7.39 \mathrm{~Hz}, 4 \mathrm{H}, \mathrm{H6}) ; 7.54$ (d, $J=7.41 \mathrm{~Hz}, 4 \mathrm{H}, \mathrm{H} 3) ; 7.59$ (d, $J=$ $6.91 \mathrm{~Hz}, 4 \mathrm{H}, \mathrm{H} 2) .{ }^{13} \mathrm{C} \mathrm{NMR}\left(\mathrm{CDCl}_{3}, \delta, \mathrm{ppm}\right):-2.30$ (C13); 116.24 (C11); 117.36 (C7); 121.24 (C10); 126.24 (C3); 128.22 (C6); 134.67 (C2); 134.89 (C5); 136.41 (C1); 141.34 (C4); 142.84 (C12), 148.38 (C9); 158.67 (C8). ${ }^{29} \mathrm{Si} \mathrm{NMR}\left(\mathrm{CDCl}_{3}, \delta, \mathrm{ppm}\right):-8.09$. Elem. anal. calcd for $\mathrm{C}_{38} \mathrm{H}_{34} \mathrm{~N}_{2} \mathrm{O}_{2} \mathrm{Si}$; (578.83): C, 78.84\%; H, 5.93\%; N, 4.84\%. Found: C, $78.81 \%$; H, 5.89\%, N, 4.81\%.

Bis(4-(4-(4-aminophenoxy)phenyl)phenyl)diphenylsilane (8). $\mathrm{Mp}=163{ }^{\circ} \mathrm{C}$ to $164{ }^{\circ} \mathrm{C}$. FT-IR $\left(\mathrm{KBr}, \nu, \mathrm{cm}^{-1}\right): 3370,3444(\mathrm{~N}-\mathrm{H})$; 3011, 3039, 3065 (C-H arom.); 1486, 1505, 1597 (C=C); 1235 (C$\mathrm{O}-\mathrm{C}) ; 1111$ (Si-Ph); 816 (arom. $p$-subst.). ${ }^{1} \mathrm{H}$ NMR $\left(\mathrm{CDCl}_{3}, \delta\right.$, ppm): 3.49 (s, 4H, H17); 6.68 (d, $J=8.61 \mathrm{~Hz}, 4 \mathrm{H}, \mathrm{H} 11) ; 6.90$ (d, $=8.62 \mathrm{~Hz}, 4 \mathrm{H}, \mathrm{H} 10) ; 6.99(\mathrm{~d}, J=8.56 \mathrm{~Hz}, 4 \mathrm{H}, \mathrm{H} 7), 7.39(\mathrm{~m}, 4 \mathrm{H}$, H15); 7.44 (m, 2H, H16); 7.52 (d, $J=8.58 \mathrm{~Hz}, 4 \mathrm{H}, \mathrm{H} 6) ; 7.56$ (d, $J$ $=7.88 \mathrm{~Hz}, 4 \mathrm{H}, \mathrm{H} 3), 7.62(\mathrm{~d}, J=6.26 \mathrm{~Hz}, 4 \mathrm{H}, \mathrm{H} 14) ; 7.64(\mathrm{~d}, J=$ $7.62 \mathrm{~Hz}, 4 \mathrm{H}, \mathrm{H} 2) .{ }^{13} \mathrm{C} \mathrm{NMR}\left(\mathrm{CDCl}_{3}, \delta, \mathrm{ppm}\right): 116.26$ (C11); 117.40 (C7); 121.27 (C10); 126.24 (C3); 127.93 (C15); 128.25 (C6); 129.64 (C16); 132.42 (C1); 134.28 (C13); 134.74 (C5); 136.41 (C14); 136.87 (C2); 141.71 (C4); 142.83 (C12); 148.41 (C9); 158.78 (C8). ${ }^{29} \mathrm{Si} \mathrm{NMR}\left(\mathrm{CDCl}_{3}, \delta, \mathrm{ppm}\right):-14.52$. Elem. anal. calcd for $\mathrm{C}_{48} \mathrm{H}_{38} \mathrm{~N}_{2} \mathrm{O}_{2} \mathrm{Si}$; (698.93): C, 82.48\%; H, 4.91\%; N, 4.01\%. Found: C, $82.42 \%$; H, $4.85 \%$, N, 3.96\%.

Bis(4-(4-formylphenyl)phenyl)di-R-silane ( $R=$ methyl (9), phenyl (10)). To a mixture of dibromo compound (1 or 2) (18.50 mmol), bis(triphenylphosphine) palladium(II) dichloride $(0.55 \mathrm{~g}, 0.784 \mathrm{mmol})$ and 4-formylyphenylboronic acid (6.935 g, $46.25 \mathrm{mmol}$ ) a solution containing $\mathrm{K}_{2} \mathrm{CO}_{3}(16.50 \mathrm{~g})$ dissolved in $126 \mathrm{~mL}$ of a water-1,4-dioxane mixture (1: $1 \mathrm{vol} / \mathrm{vol})$ was added; then, the mixture was heated at $85{ }^{\circ} \mathrm{C}$ for 12 hours. Then, water was added, and a dark brown solid was obtained which was filtered under vaccum. This product was treated with THF, and the remaining solids were removed by filtration through celite. The obtained solution was dried and concentrated to obtain a black solid, which was purified by column chromatography (silica/petroleum ether-chloroform, 1:1 vol/vol) and subsequent recrystallization from ethyl acetate. Following this procedure, $\mathbf{9}$ and $\mathbf{1 0}$ were obtained as white solids in yields of $56 \%$ and $51 \%$, respectively.<smiles>O=Cc1ccc(-c2ccc(I)cc2)cc1</smiles><smiles>[R][SiH](C)CCCCC</smiles><smiles>[R]c1ccc(-c2ccc(C=O)cc2)cc1</smiles>

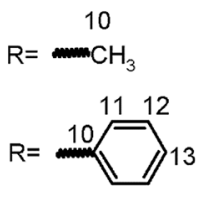

Bis(4-(4-formylphenyl)phenyl)dimethylsilane (9). $\mathrm{Mp}=$ $230{ }^{\circ} \mathrm{C}$ to $233{ }^{\circ} \mathrm{C}$. FT-IR (KBr, $\left.\nu, \mathrm{cm}^{-1}\right): 3013,3030,3047,3070$ (C-H arom.); 2957 (C-H aliph.); 2737, 2823 (C-H ald.); 1694 (C= O); 1604, 1572 (C=C); $1387\left(\mathrm{Si}-\mathrm{CH}_{3}\right) ; 1166,1111$ (Si-Ph); 813 (arom. $p$-subst.). ${ }^{1} \mathrm{H}$ NMR (DMSO- $d_{6}, \delta$, ppm): 0.64 (s, 6H, H10); $7.70(\mathrm{~d}, J=8.07 \mathrm{~Hz}, 4 \mathrm{H}, \mathrm{H} 2) ; 7.74(\mathrm{~d}, J=8.04 \mathrm{~Hz}, 4 \mathrm{H}, \mathrm{H} 3) ; 7.86$ (d, $J=8.16 \mathrm{~Hz}, 4 \mathrm{H}, \mathrm{H} 6) ; 7.97$ (d, $J=8.23 \mathrm{~Hz}, 4 \mathrm{H}, \mathrm{H} 7) ; 10.08$ (s, $2 \mathrm{H}, \mathrm{H} 9$ ). ${ }^{13} \mathrm{C}$ NMR (DMSO- $\left.d_{6}, \delta, \mathrm{ppm}\right):-3.48$ (C10); 125.72 (C3); 126.55 (C6); 129.08 (C7); 133.83 (C2); 134.98 (C8); 137.55 (C1), 139.12 (C4); 145.14 (C5); 191.12 (C9). ${ }^{29} \mathrm{Si}$ NMR (DMSO- $d_{6}, \delta$, ppm): -7.85. Elem. anal. calcd for $\mathrm{C}_{28} \mathrm{H}_{24} \mathrm{O}_{2} \mathrm{Si}$; (420.61): C, 79.95\%; H, 5.76\%. Found: C, 79.91\%; H, 5.70\%.

Bis(4-(4-formylphenyl)phenyl)diphenylsilane (10). $\mathrm{Mp}=$ $272{ }^{\circ} \mathrm{C}$ to $275{ }^{\circ} \mathrm{C}$. FT-IR $\left(\mathrm{KBr}, \nu, \mathrm{cm}^{-1}\right): 3015,3049,3068(\mathrm{C}-\mathrm{H}$ arom.); 2733, 2821 (C-H ald.); $1700(\mathrm{C}=\mathrm{O}) ; 1572,1604(\mathrm{C}=\mathrm{C})$; 1111, 1429 (Si-Ph); 810 (arom. $p$-subst.). ${ }^{1} \mathrm{H}$ NMR $\left(\mathrm{CDCl}_{3}, \delta\right.$, ppm): 7.42 (d, $J=7.17 \mathrm{~Hz}, 4 \mathrm{H}, \mathrm{H} 12) ; 7.48$ (m, 2H, H13); 7.63 (d, $J$ $=7.28 \mathrm{~Hz}, 4 \mathrm{H}, \mathrm{H} 11) ; 7.67$ (d, $J=7.79 \mathrm{~Hz}, 4 \mathrm{H}, \mathrm{H} 3) ; 7.72(\mathrm{~d}, J=$ $7.64 \mathrm{~Hz}, 4 \mathrm{H}, \mathrm{H} 2) ; 7.78$ (d, $J=7.88 \mathrm{~Hz}, 4 \mathrm{H}, \mathrm{H} 6) ; 7.96$ (d, $J=$ $7.85 \mathrm{~Hz}, 4 \mathrm{H}, \mathrm{H} 7) ; 10.06$ (s, 2H, H9). ${ }^{13} \mathrm{C} \mathrm{NMR}\left(\mathrm{CDCl}_{3}, \delta, \mathrm{ppm}\right)$ : 126.84 (C3); 127.72 (C6); 128.09 (C12); 129.92 (C13); 130.32 (C7); 133.60 (C10); 134.50 (C1); 135.44 (C8); 136.38 (C11); 137.05 (C2); 140.86 (C4); 146.81 (C5); 191.83 (C9). ${ }^{29} \mathrm{Si} \mathrm{NMR}\left(\mathrm{CDCl}_{3}, \delta, \mathrm{ppm}\right)$ : -14.36. Elem. anal. calcd for $\mathrm{C}_{38} \mathrm{H}_{28} \mathrm{O}_{2} \mathrm{Si}$; (544.75): C, 83.78\%; H, 5.19\%. Found: C, 83.72\%; H, 5.13\%.

\section{Silylated oligomeric poly(ether-azomethine)s synthesis}

A mixture of dialdehyde (9 or 10), anhydrous calcium chloride, $p$-toluenesulfonic acid $(p$-TsOH) and anhydrous DMAc was placed under a nitrogen atmosphere with vigorous stirring and heated until reflux. When the dialdehyde was dissolved, a solution containing the diamine (7 or 8) in anhydrous DMAc prepared under nitrogen flow was slowly added; the reaction was then maintained under reflux for 24 hours. Then, the mixture was added to methanol under stirring and the obtained solid was filtered, extensively washed with hot methanol, and dried at $100{ }^{\circ} \mathrm{C}$ under vacuum for 24 hours.

PAM-I. Yield: 79\%, FT-IR (KBr, $\left.\nu, \mathrm{cm}^{-1}\right): 3063,3032,3013$ (C$\mathrm{H}$ arom.); 2954 (C-H aliph.); 2877 (CO-H); 1698 (C=O); 1624 $(\mathrm{C}=\mathrm{N}) ; 1603$, 1496, $1487(\mathrm{C}=\mathrm{C}) ; 1244(\mathrm{C}-\mathrm{O}-\mathrm{C}) ; 1114(\mathrm{Si}-\mathrm{Ph})$; $872\left(\mathrm{Si}-\mathrm{CH}_{3}\right) ; 810$ (arom. $p$-subst.). ${ }^{1} \mathrm{H}$ NMR (DMAc, acetone- $d_{6}$ internal reference (int. ref.), $\delta$, ppm): 0.47 (m, 12H, H22, H23); 7.14 (d, $J=7.25 \mathrm{~Hz}, 4 \mathrm{H}, \mathrm{H} 7), 7.29$ (m, 4H, H10); 7.55 (m, 8H, H11, H20); 7.61-7.84 (m, 16H, H2, H3, H6, H19); 7.97 (m, 8H, H15, H16); 8.80 (s, 2H, H13). ${ }^{13} \mathrm{C}$ NMR (DMAc, acetone- $d_{6}$ int. ref., $\delta$, ppm): -3.333; -3.329 (C22, C23); 121.55 (C7); 122.82 (C10); 126.36 (C3); 126.46 (C19); 127.46, 127.56, 127.79 (C6, C11, C16); 129.04 (C15); 132.13 (C5); 133.21; 133.22 (C2, C20); 133.61, 


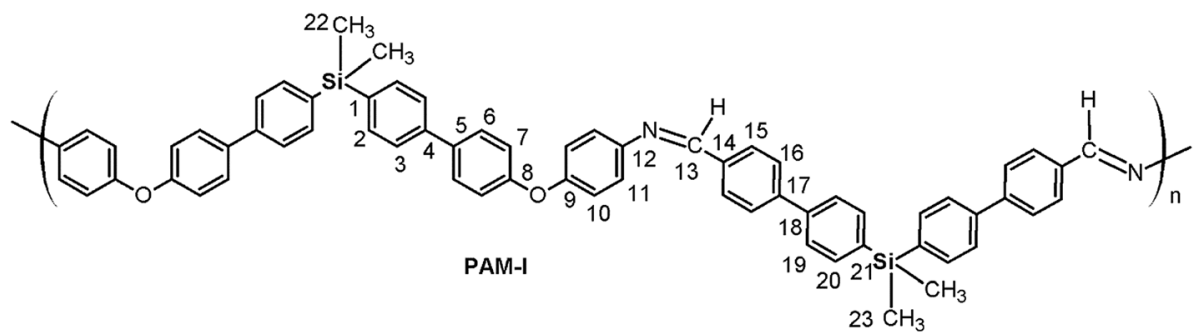

133.70, 134.70, 135.63 (C1, C14, C18, C21); 139.31 (C4); 144.34 (C17); 148.94 (C12); 154.28 (C9); 156.95 (C8); 164.05 (C13). ${ }^{29} \mathrm{Si}$ NMR (DMAc, acetone- $d_{6}$ int. ref., $\left.\delta, \mathrm{ppm}\right)$ : -9.82 (diamino moiety); -9.79 (dialdehyde moiety). $d_{6}$ int. ref., $\left.\delta, \mathrm{ppm}\right): 0.66$ (s, 6H, H26); 7.17 (m, 8H, H7, H10); 7.40-5.56 (m, 10H, H11, H24, H25); 7.63 (m, 4H, H23); 7.68 (m, $4 \mathrm{H}, \mathrm{H} 2)$; 7.74 (m, 4H, H20), 7.75-7.87 (m, 12H, H3, H6, H19); 7.90 (m, 4H, H16), 8.10 (m, 4H, H15); 8.80 (s, 2H, H13). ${ }^{13} \mathrm{C}$ NMR (DMAc, acetone- $d_{6}$ int. ref., $\delta, \mathrm{ppm}$ ): -2.67 (C26), 119.19 (C7),

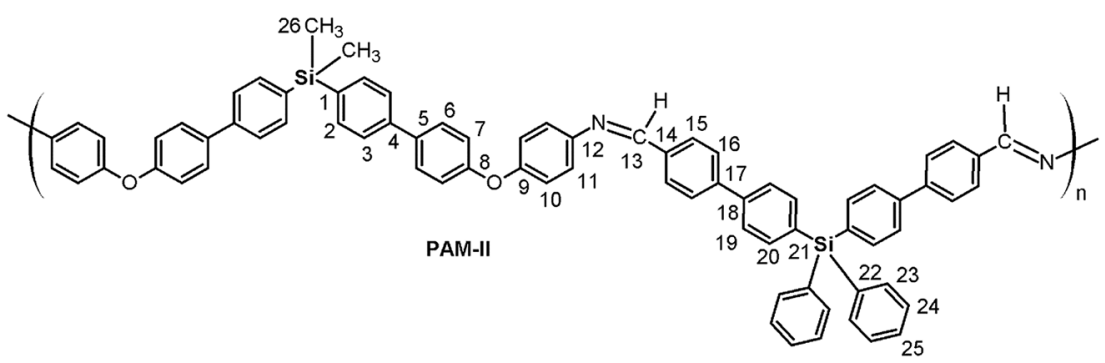

PAM-II. Yield: 86\%, FT-IR (KBr, $\left.\nu, \mathrm{cm}^{-1}\right)$ : 3065, 3043, 3030, 3013 (C-H arom.); 2951 (C-H aliph.); 2878 (CO-H); $1700(\mathrm{C}=\mathrm{O})$; $1624(\mathrm{C}=\mathrm{N}) ; 1603,1495,1486(\mathrm{C}=\mathrm{C}) ; 1240(\mathrm{C}-\mathrm{O}-\mathrm{C}) ; 1112$ (Si$\mathrm{Ph}) ; 871$ (Si-CH$) ; 811$ (arom. $p$-subst). ${ }^{1} \mathrm{H}$ NMR (DMAc, acetone$d_{6}$ int. ref., $\left.\delta, \mathrm{ppm}\right): 0.63$ (s, 6H, H26); 7.15 (m, 8H, H7, H10); 7.47 (d. $J=8.11 \mathrm{~Hz}, 4 \mathrm{H}, \mathrm{H} 11$ ); 7.53 (m, 10H, H24, H25); 7.587.81 (m, 20H, H2, H3, H6, H20, H23); 7.94 (m, 8H, H16, H19); 8.13 (m, 4H, H15); 8.82 (s, 2H, H13). ${ }^{13} \mathrm{C}$ NMR (DMAc, acetone$d_{6}$ int. ref., $\delta, \mathrm{ppm}$ ): -2.56 (C26); 119.15 (C7); 120.15 (C10); 123.28 (C11); 126.39 (C3); 127.02 (C19); 127.59 (C16); 128.65 (C24); 128.76 (C6); 129.72 (C15); 130.53 (C25); 133.95, 134.02, 134.13 (C14, C21, C22); 135.09 (C2); 135.83 (C5); 136.50 (C23); 136.99 (C1); 137.22 (C20); 141.00 (C4); 141.47 (C18); 143.09 (C17); 147.84 (C12); 155.59 (C9); 157.63 (C8); 159.62 (C13). ${ }^{29} \mathrm{Si}$ NMR (DMAc, acetone- $d_{6}$ int. ref., $\delta$, ppm): -15.34 (dialdehyde moiety); -9.11 (diamine moiety).
120.20 (C10); 123.29 (C11); 126.62 (C3); 126.80 (C19); 127.54 (C16); 128.61 (C24); 128.87 (C6); 129.71 (C15); 130.52 (C25); 132.88 (C1); 134.38 (C22); 135.20 (C20); 135.51 (C5); 136.49 (C23); 137.16 (C2); 138.10 (C21); 140.35 (C18); 140.88 (C14); 141.63 (C4); 143.42 (C17); 147.94 (C12); 155.54 (C9); 157.84 (C8); 159.71 (C13). ${ }^{29} \mathrm{Si}$ NMR (DMAc, acetone- $d_{6}$ int. ref., $\delta, \mathrm{ppm}$ ): -15.35 (diamine moiety); -8.33 (dialdehyde moiety).

PAM-IV. Yield: 68\%, FT-IR (KBr, $\left.\nu, \mathrm{cm}^{-1}\right): 3066,3044,3022$, 3013 (C-H arom.); 2876, $2732(\mathrm{CO}-\mathrm{H}) ; 1700(\mathrm{C}=\mathrm{O}) ; 1623(\mathrm{C}=$ $\mathrm{N})$; 1603, 1496, 1486 (C=C); 1238 (C-O-C); 1110 (Si-Ph); 813 (arom. $p$-subst). ${ }^{1} \mathrm{H}$ NMR (DMAc, acetona- $d_{6}$ int. ref., $\delta, \mathrm{ppm}$ ): 7.18 (m, 8H, H7, H10); 7.47 (m, 16H, H11, H24, H25, H28, H29); 7.65 (m, 12H, H2, H23, H27); 7.75 (m, 4H, H20); 7.82 (m, 8H, H3, H6); 7.95 (m, 8H, H16, H19); 8.13 (d, $J=6.13 \mathrm{~Hz}, 4 \mathrm{H}, \mathrm{H} 15)$, 8.82 (s, 2H, H13). ${ }^{13} \mathrm{C}$ NMR (DMAc, acetone- $d_{6}$ int. ref., $\delta, \mathrm{ppm}$ ): 119.19 (C7); 120.19 (C10); 123.29 (C11); 126.62 (C3); 127.04

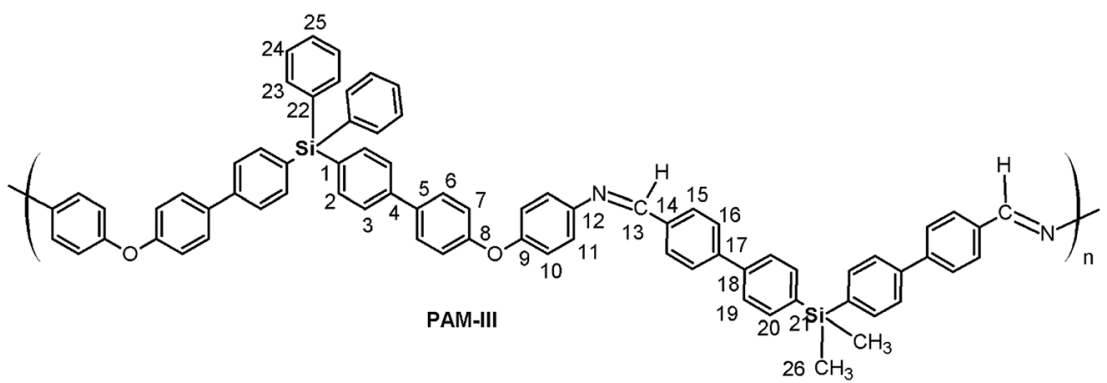

PAM-III. Yield: 65\%, FT-IR (KBr, $\left.\nu, \mathrm{cm}^{-1}\right)$ : 3067, 3033, 3014 (C-H arom.); 2953 (C-H aliph.); 2876, 2732 (CO-H); $1700(\mathrm{C}=\mathrm{O})$; $1623(\mathrm{C}=\mathrm{N})$; 1603, 1496, $1486(\mathrm{C}=\mathrm{C}) ; 1239$ (C-O-C); 1112 (Si$\mathrm{Ph}) ; 872$ ( $\left.\mathrm{Si}-\mathrm{CH}_{3}\right) ; 808$ (arom. $p$-subst). ${ }^{1} \mathrm{H}$ NMR (DMAc, acetone-
(C19); 127.60 (C16); 128.60 (C24); 128.67 (C28); 128.86 (C6); 129.74 (C15); 130.34, 130.52 (C25, C29); 132.87 (C1); 134.03 (C21); 134.35, 134.38 (C22, C26); 135.52 (C5), 136.49 (C23, C27); 137.16 (C2); 137.23 (C20); 140.96 (C18); 141.49 (C14); 141.62 


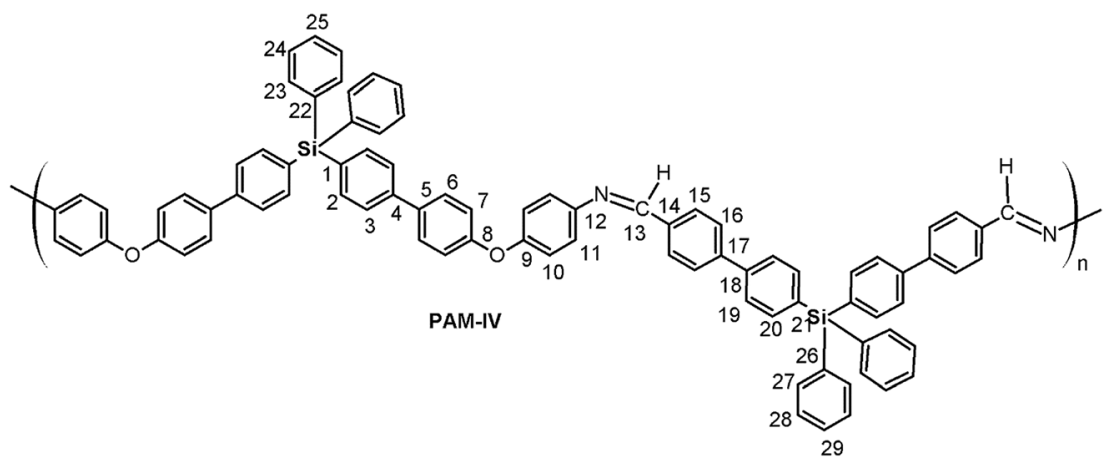

(C4); 143.11 (C17); 147.90 (C12); 155.56 (C9); 157.82 (C8); 159.66 (C13). ${ }^{29}$ Si NMR (DMAc, acetone- $d_{6}$ int. ref., $\left.\delta, \mathrm{ppm}\right):-15.33$; -15.31 .

\section{Results and discussion}

\section{Monomer synthesis}

Both monomers use dibromo derivatives ( 1 or 2 ) as common precursors (bis(4-bromophenyl)di-R-silanes); these were directly obtained by means of controlled mono-lithiation of 1,4-dibromobenzene and then reacted with the respective dichlorosilanes (Fig. 1, step a). In this last step, the nucleophilic substitution on the central silicon atom is favored because the $\mathrm{Si}-\mathrm{X}$ distances $(\mathrm{X}=\mathrm{Cl}, \mathrm{C}$ ) in the organo-silane compounds are longer than the $\mathrm{C}-\mathrm{X}$ bond distances. Thus, the steric hindrance for $\mathrm{S}_{\mathrm{N}} 2$ attack was negligible, and yields of over $70 \%$ were obtained.

Elemental analysis, FT-IR spectroscopy and NMR spectroscopy techniques, including COSY, HMQC, and HMBC, were employed to establish the structures of the dibromo compounds and of all the precursors and monomers. In this case, the main IR bands are associated with the $\mathrm{C}-\mathrm{Si}$ bonds at 1373 to $1427 \mathrm{~cm}^{-1}$ and $1253 \mathrm{~cm}^{-1}$ according to the nature of the carbon atom, aromatic or aliphatic, respectively, and the $\mathrm{C}-\mathrm{Br}$ stretching band observed at approximately $510 \mathrm{~cm}^{-1}$. The ${ }^{1} \mathrm{H}$ and ${ }^{13} \mathrm{C}$ NMR spectra clearly showed displacement due to the high magnetic fields exerted by the silicon atoms over their environment. Thus, the $\mathrm{H}$ and $\mathrm{C}$ nuclei of the methyl group of $\mathbf{1}$ appear at $0.44 \mathrm{ppm}$ and $-2.42 \mathrm{ppm}$, respectively. Also, the aromatic carbon atoms bonded directly to the silicon atom were observed at high field. This effect can be observed in the spectra of all the silylated precursors and monomers.

The biphenyl moieties were incorporated into the monomers and, therefore, the final polymers by Suzuki coupling reactions. Thus, dibromo compounds $\mathbf{1}$ or $\mathbf{2}$ were reacted with the corresponding boronic acid derivatives to produce the dialdehyde monomers 9 and 10 (Fig. 1, step e) and the diphenols 3 and 4 (Fig. 1, step b). The FT-IR spectra of the latter compounds showed the characteristic phenolic $\mathrm{O}-\mathrm{H}$ band between 3500 and $3400 \mathrm{~cm}^{-1}$. Likewise, the ${ }^{1} \mathrm{H}$ NMR spectra evidenced this function, with a narrow singlet at $9.59 \mathrm{ppm}$ for 3 and a broad singlet centered at $5.11 \mathrm{ppm}$ for $\mathbf{4}$. In the latter case, the electronic contribution of the lateral phenyl groups bonded to the silicon atom probably promotes electronic shielding of this nucleus.

By means of arylation of the oxygen atoms using a potassium carbonate aqueous solution as a basic medium and 1-fluoro-4nitrobenzene, the dinitro derivatives $\mathbf{5}$ and $\mathbf{6}$ were prepared with yields of $88 \%$ and $74 \%$, respectively (Fig. 1, step c). For

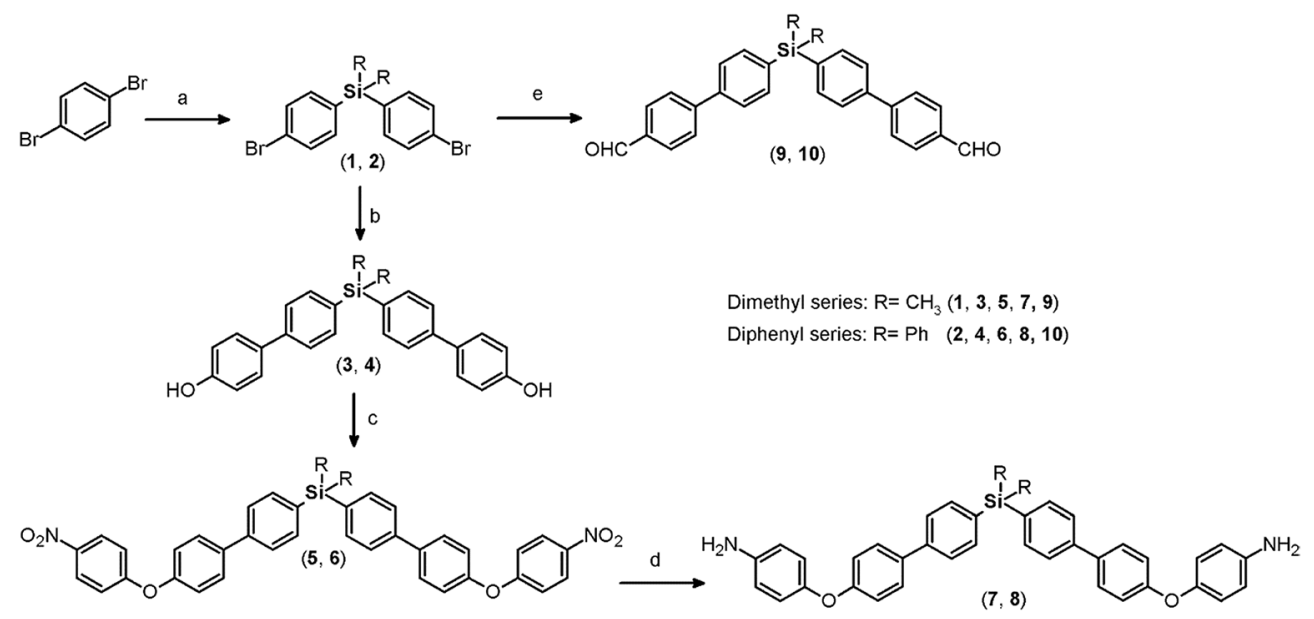

Fig. 1 Synthesis of monomers containing silicon and biphenyl moieties. (a) (1) $n$-BuLi, $\mathrm{Et}_{2} \mathrm{O}$ anh., $0^{\circ} \mathrm{C}$. (2) $\mathrm{R}_{2} \mathrm{SiCl}_{2}\left(\mathrm{R}=\mathrm{CH}_{3}, \mathrm{Ph}\right), \mathrm{Et}{ }_{2} \mathrm{O}$ anh.; (b) 4 hydroxyphenylboronic acid, $\mathrm{Pd}\left(\mathrm{PPh}_{3}\right)_{2} \mathrm{Cl}_{2}, \mathrm{~K}_{2} \mathrm{CO}_{3}$ (aq.) $2 \mathrm{M}, 1,4$-dioxane, $85^{\circ} \mathrm{C}$; (c) 1-fluoro-4-nitrobenzene, $\mathrm{K}_{2} \mathrm{CO}_{3}, \mathrm{DMF}^{\circ} 60^{\circ} \mathrm{C}$; (d) $\mathrm{H}_{2} \mathrm{~N}$ - $\mathrm{NH} \mathrm{H}_{2}$ (80\%), $\mathrm{Pd} / \mathrm{C}$ (10\%), THF, reflux; (e) 4-formylphenylboronic acid, $\mathrm{Pd}\left(\mathrm{PPh}_{3}\right)_{2} \mathrm{Cl}_{2}, \mathrm{~K}_{2} \mathrm{CO}_{3}$ (aq.) $2 \mathrm{M}, 1,4$-dioxane, $85^{\circ} \mathrm{C}$. 
these compounds, IR bands originating from the $\mathrm{NO}_{2}$ groups were observed at 1518 and $1344 \mathrm{~cm}^{-1}$ and from the stretching ether linkage $(\mathrm{C}-\mathrm{O}-\mathrm{C})$ at about $1236 \mathrm{~cm}^{-1}$. The aromatic zones of the NMR spectra of both dinitro compounds showed new signals for the $\mathrm{Ph}-\mathrm{NO}_{2}$ moieties, like an $\mathrm{AB}$ system, at 6.68 and $6.90 \mathrm{ppm}$, without evidence of the signals at about 9.6 and $5.1 \mathrm{ppm}$ attributed to the hydroxylic groups of the respective precursors.

The reduction of the $\mathrm{NO}_{2}$ groups to produce diamine monomers 7 and $\mathbf{8}$ was carried out following a classic procedure which involves the use of hydrazine and Pd/C (Fig. 1, step d). In both cases, chromatographic techniques were employed to purify the products. In the case of diamine 7 , additional recrystallization from acetonitrile was required. The amino group was easily detected in the FT-IR spectra through two stretching bands between 3340 and $3450 \mathrm{~cm}^{-1}$ (Fig. 2). Also, the ${ }^{1} \mathrm{H}$ NMR spectra reported evidence of the reduction. A narrow singlet with an integration of $4 \mathrm{H}$ associated with $\mathrm{NH}_{2}$ groups was observed at $3.57 \mathrm{ppm}$ for 7 ; meanwhile, for diamine 8, a broad and flat singlet centered at $3.49 \mathrm{ppm}$ also showed an integration of $4 \mathrm{H}$ (Fig. 3). Likewise, in these spectra and the ${ }^{13} \mathrm{C}$ spectra, the aromatic zone was altered when $\mathrm{NH}_{2}$ replaced $\mathrm{NO}_{2}$ due to the contrasting electronic effects mainly exerted on the adjacent phenyl groups.

On the other hand, for the silicon-containing dialdehydes, the FT-IR spectra showed the characteristic stretching band for carbonyl groups close to $1700 \mathrm{~cm}^{-1}$, independently of the $\mathrm{R}$ group bonded to the silicon atom (Fig. 2). The ${ }^{1} \mathrm{H}$ NMR spectra demonstrated the proton of the aldehyde group at $10 \mathrm{ppm}$, while the ${ }^{13} \mathrm{C}$ NMR spectra showed the carbonylic carbon at 191 ppm (Fig. 4).

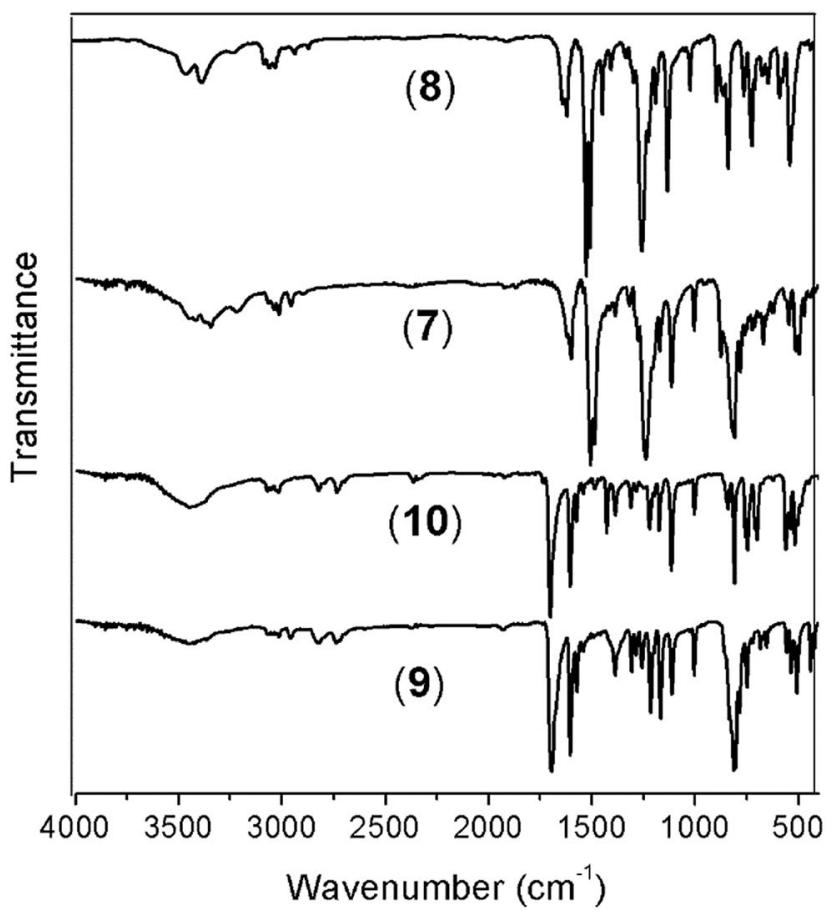

Fig. 2 FT-IR spectra (vertically shifted) of the silylated monomers Diamines 7 and 8; dialdehydes 9 and 10 .
In all cases (precursors and monomers), the ${ }^{29} \mathrm{Si}$ NMR spectra showed two different signals. For the dimethyl series (1, 3, 5, 7 and 9), a band could be observed between $-7.00 \mathrm{ppm}$ and -8.64 ppm; meanwhile, for the diphenyl series (2, 4, 6, 8 and 10), this signal was observed close to $-14 \mathrm{ppm}$. These values are associated with the $-\mathrm{Ph}-\mathrm{Si}\left(\mathrm{CH}_{3}\right)_{2}-\mathrm{Ph}-$ and $-\mathrm{Ph}-\mathrm{Si}(\mathrm{Ph})_{2}-\mathrm{Ph}-$ moieties, respectively, and can be explained according to the particular electronic effects of the methyl and phenyl groups. This phenomenon has been previously observed in several monomer and polymer families synthesized by our research group. ${ }^{31-33}$

\section{Oligomeric poly(ether-azomethine)s synthesis and spectroscopic characterization}

Four new silylated oligomeric poly(ether-azomethine)s were prepared through a polycondensation procedure in DMAc solution and under an inert atmosphere. The $\mathrm{pH}$ of the reaction was adjusted to 5.0 by adding $p$-TsOH, and anhydrous $\mathrm{CaCl}_{2}$ was employed to capture water, which is the byproduct of the dehydration of the carbonylamine intermediate (Fig. 5). The solutions obtained after heating at $180{ }^{\circ} \mathrm{C}$ over $24 \mathrm{~h}$ were added to methanol, precipitating light beige solids in all cases. The yields were between $65 \%$ and $86 \%$.

The structural characterization of the samples was performed using spectroscopic techniques. $\mathrm{KBr}$ pellets were prepared, and the IR main bands were assigned (see Experimental section). Thus, the stretching $\mathrm{C}=\mathrm{N}$ of the imine group was identified at $1623 \mathrm{~cm}^{-1}$. In all cases, a $\mathrm{C}=\mathrm{O}$ band of variable intensity was observed close to $1700 \mathrm{~cm}^{-1}$; this is not attributed to residual monomer due to the exhaustive washing procedure of the samples after the isolation step. Additionally, TLC analysis using $\mathrm{CHCl}_{3}$ as the mobile phase did not show a monomer signal, indicating that the IR band is associated with terminal groups of short chains (oligomers). In this sense, the presence of $\mathrm{NH}_{2}$ chain terminal groups is not clearly defined in the spectra, probably due to their lower intensity in relation to the carbonyl band.

Fig. 6 shows a representative FT-IR spectrum of the obtained materials and the definition of the $d_{\mathrm{CO}}$ and $d_{\mathrm{CN}}$ distances, which are associated with the intensity of the carbonyl and imine bands, respectively. Using these values, it is possible to establish the aldehyde average contribution parameters inside the chains (AC):

$$
\mathrm{AC}=d_{\mathrm{CO}} /\left(d_{\mathrm{CO}}+d_{\mathrm{CN}}\right)
$$

Thus, the samples PAM-I to PAM-IV showed values of 0.28, $0.36,0.52$ and 0.51 , respectively. According to these data, PAM-I and PAM-II have lower proportions of CHO in their chains and are the longest oligomers. On the other hand, PAM-III and PAMIV show similar aldehyde contributions and have comparable lengths.

Initially, DMSO- $d_{6}$ was used as the solvent to perform structural characterization of the samples. The resolution of the obtained NMR spectra was inadequate; for this reason, the samples were analyzed in DMAc solution using a capillary with acetone- $d_{6}$ as an internal reference. In this sense, the complexity of the aromatic zone in the NMR spectra was resolved with the aid of DEPT- $135^{\circ}$ and 2D NMR (COSY, HMQC, and HMBC) 

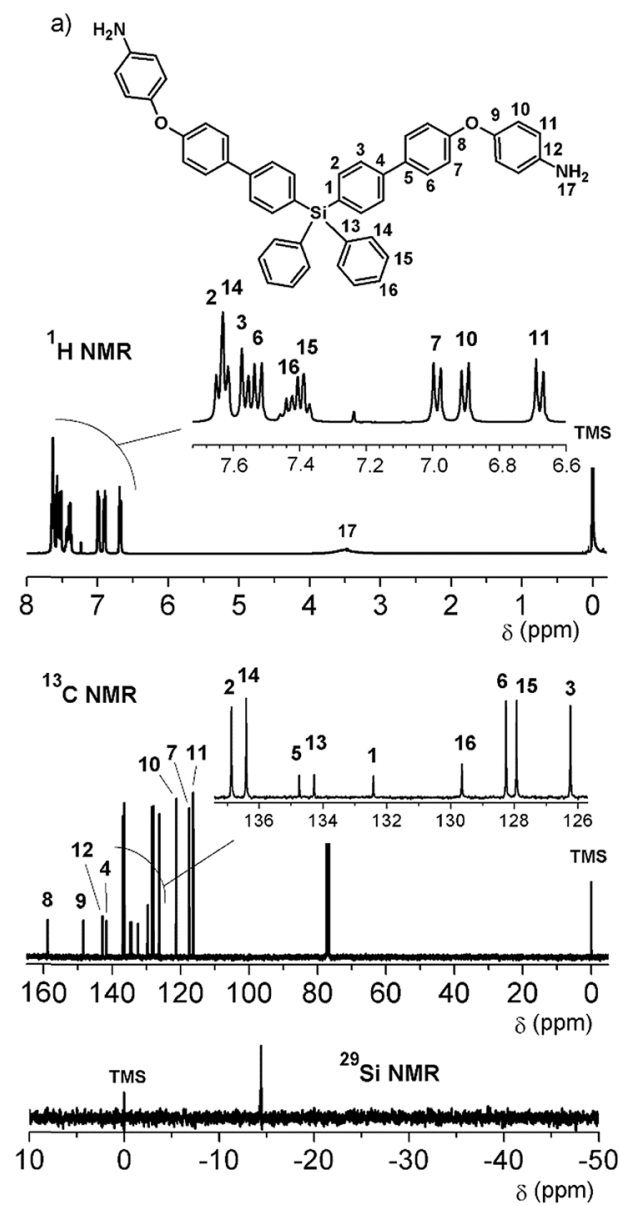

b)
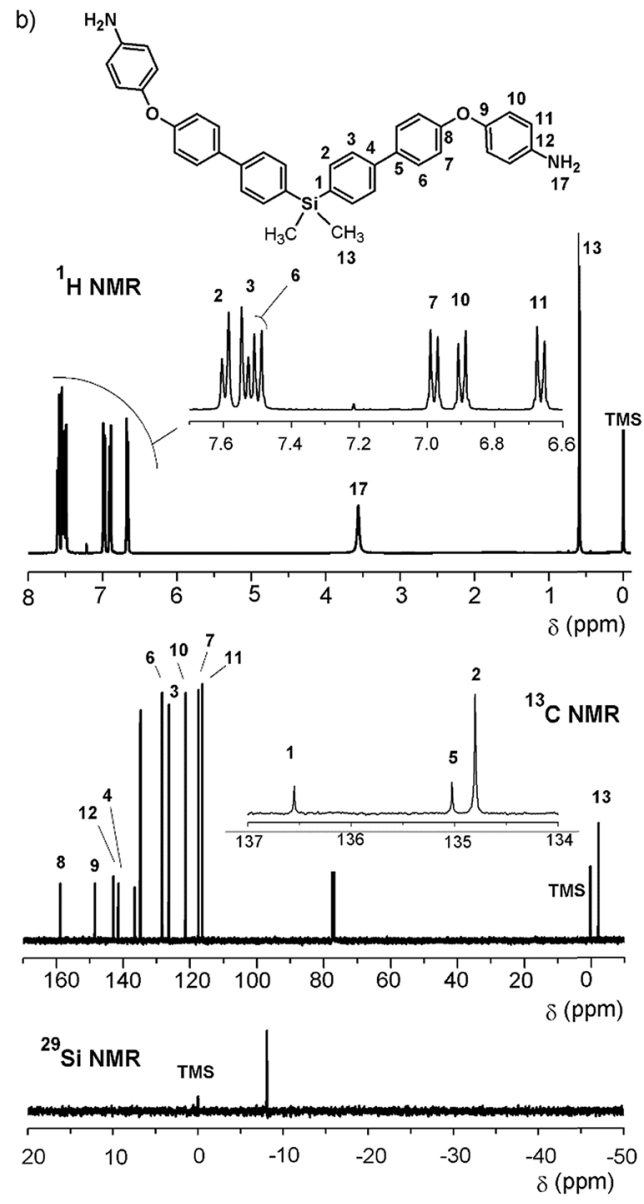

Fig. 3 NMR spectra $\left(\mathrm{CDCl}_{3}\right)$ of diamines 7 (right) and 8 (left).

analysis, enabling correct assignment of the signals. As an example, representative 2D NMR and ${ }^{29} \mathrm{Si}$ NMR spectra of PAMII are shown in Fig. 7.

In all the ${ }^{1} \mathrm{H}$ NMR spectra, a singlet at $\sim 8.8 \mathrm{ppm}$ was observed, while the ${ }^{13} \mathrm{C}$ NMR analyses evidenced a signal between 160 and $164 \mathrm{ppm}$, corroborating the formation of imine groups $(-\mathrm{CH}=\mathrm{N}-)$.

\section{Solubility, inherent viscosity and molecular weight}

The samples were tested for their ability to form solutions with several solvents; a concentration of $5 \mathrm{mg} \mathrm{mL} \mathrm{m}^{-1}$ at $19{ }^{\circ} \mathrm{C}$ was used, and the time to dissolve each sample with sporadic stirring of similar intensity was recorded. The behavior was classified as soluble/time, partially soluble and insoluble. For these last two classifications, the samples were heated at $40{ }^{\circ} \mathrm{C}$ for 24 hours. The results are shown in Table 1.

PAM-I was soluble in DMAc in $>180$ minutes, while in conc. $\mathrm{H}_{2} \mathrm{SO}_{4}$, it dissolved in less time (40 minutes). The other samples showed higher solubility in common polar organic solvents. Probably, the lower volumes of the four methyl groups bonded to the silicon atoms in PAM-I promote large packing forces between the chains; this results in $\pi$-stacking interactions, decreasing the solubility. In this sense, the ether linkages promoted by the diamine moiety and the corresponding flexibility of the chains did not increase the solubility of the sample. Among the samples, PAM-I shows the highest inherent viscosity value (Table 1); this is also associated with higher average size of the chains (see the GPC results) and, therefore, with lower solubility. At the tested concentration, all the samples were partially soluble in DMSO and insoluble in THF and $\mathrm{CHCl}_{3}$.

In general terms, PAM-II and PAM-III, whose repetitive units are structural isomers, showed solubility in DMAc and $m$-cresol; this is probably due to the introduction of bulky phenyl groups in one of the silicon atoms and therefore to diminished packing forces relative to those operating in PAM-I. On the other hand, the average molecular weights of these samples are lower than that of PAM-I. The structural effect is more evident in PAM-IV, in which all the lateral groups are phenyl rings. This sample, with an average length-chain similar to that of PAM-III, was the only one that showed solubility in DMF under the tested conditions. It is clear that the molecular weight and the structural composition of the repetitive units of the samples strongly affected the solubility behavior, where the possibility to establish interactions between the chains was limited or favored according to the volume of the groups bonded to the silicon atoms.

Additionally, a modification to the solubility test was developed by adding $\operatorname{LiBr}(0.05 \mathrm{M})$ to dilute polymer-DMF systems 
Paper

View Article Online

RSC Advances

a)

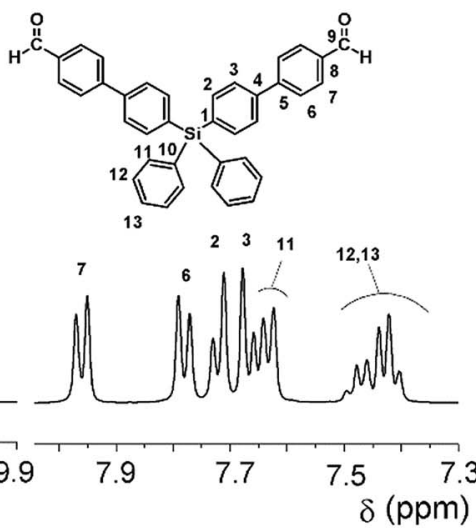

b)
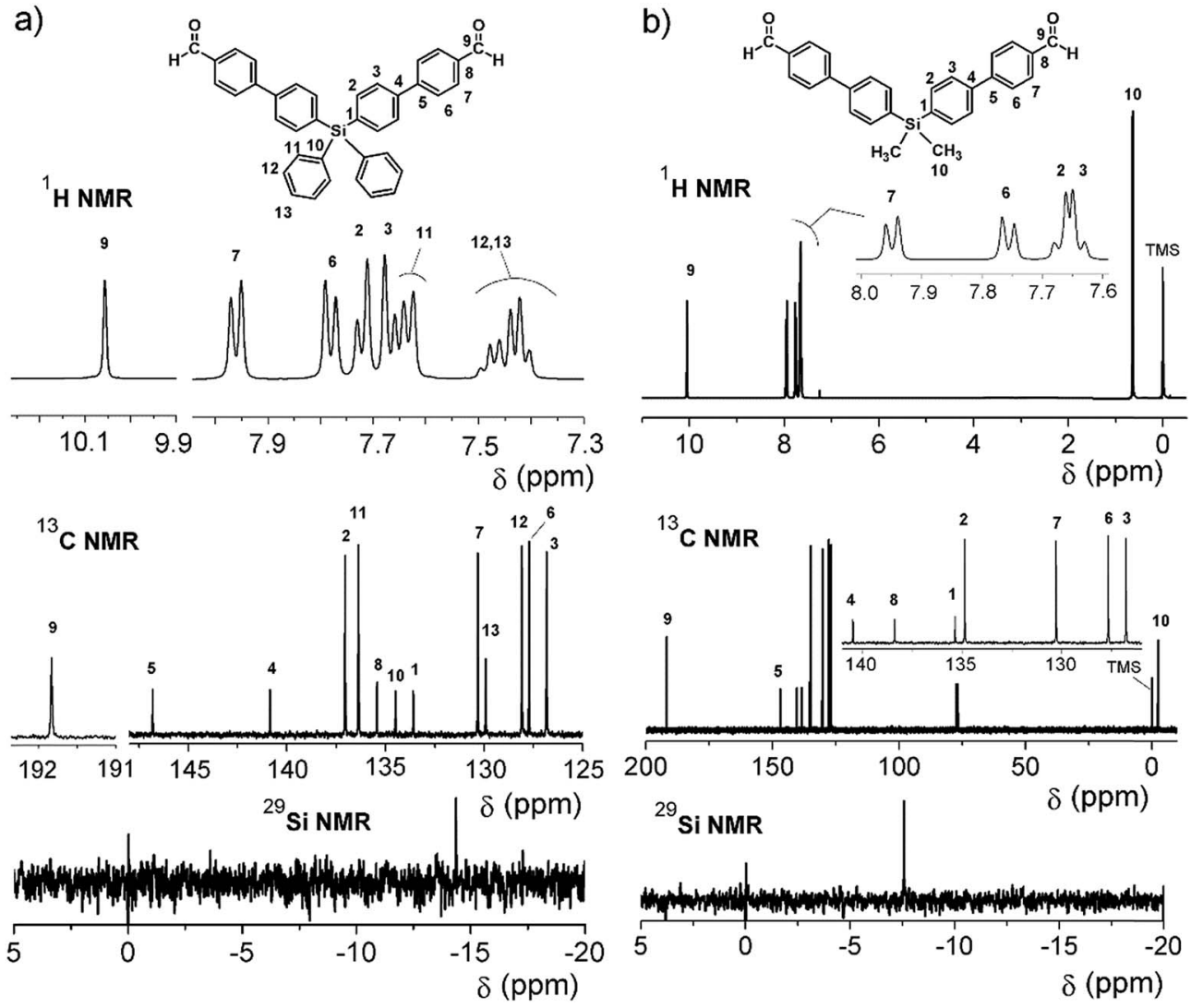

Fig. 4 NMR spectra of the dialdehydes $9\left(\mathrm{DMSO}-d_{6}\right)$ (right) and $10\left(\mathrm{CDCl}_{3}\right)$ (left).
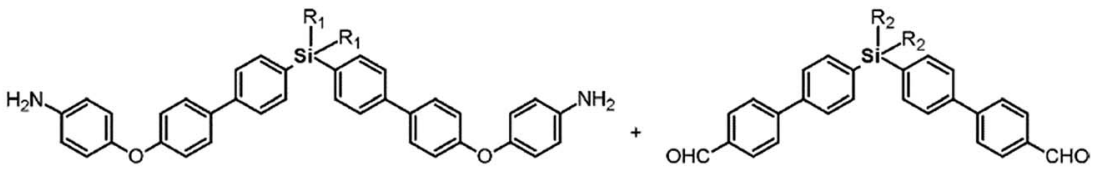

8

$p-\mathrm{TsOH}$

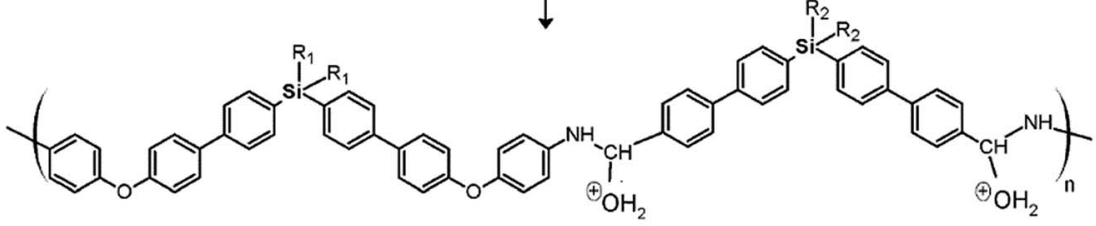

poly(carbonylamine)

$\uparrow \mid-\mathrm{H}_{2} \mathrm{O}$

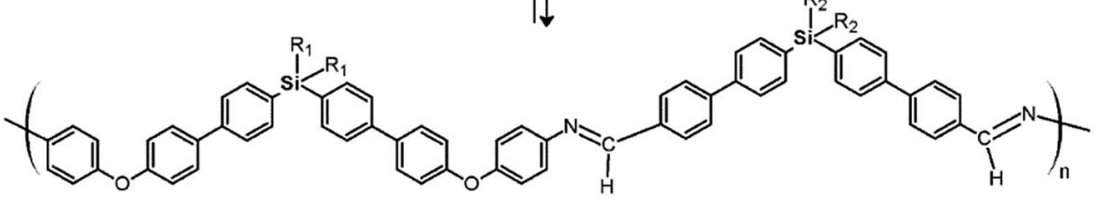

PAM-I: $\mathrm{R}^{1}=\mathrm{R}^{2}=\mathrm{CH}_{3} \quad$ PAM-III: $\mathrm{R}^{1}=\mathrm{Ph}, \mathrm{R}^{2}=\mathrm{CH}_{3}$

PAM-II: $R^{1}=C_{3}, R^{2}=P h \quad$ PAM-IV: $R^{1}=R^{2}=P h$

Fig. 5 Synthesis of silylated oligomeric poly(ether-azomethines).

This journal is (c) The Royal Society of Chemistry 2018

RSC Adv., 2018, 8, 1296-1312 | 1305 


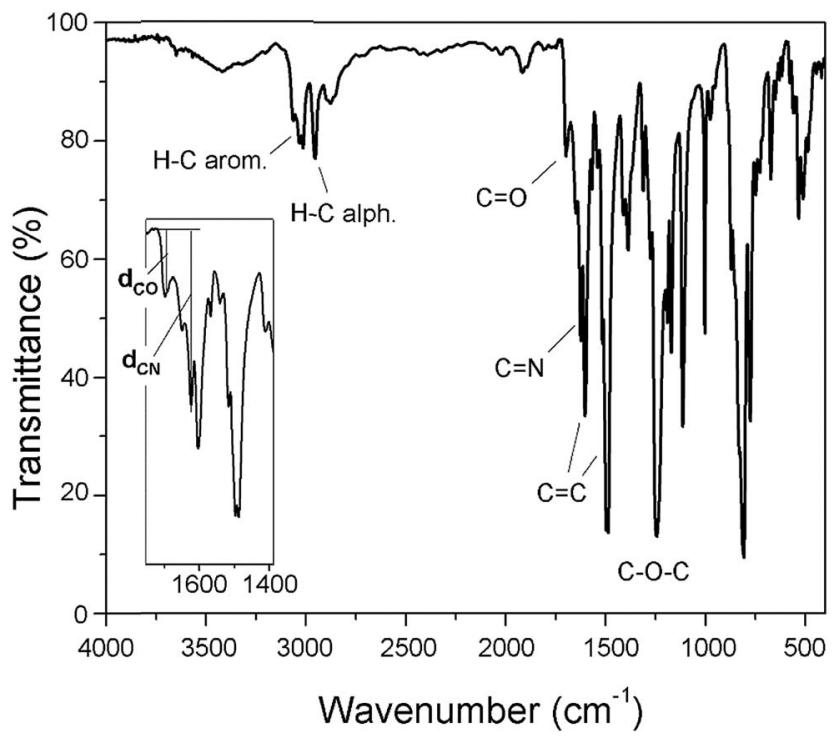

Fig. 6 The FT-IR spectrum of PAM-I with details regarding the stretching of the $\mathrm{C}=\mathrm{O}$ and $\mathrm{C}=\mathrm{N}$ bands.
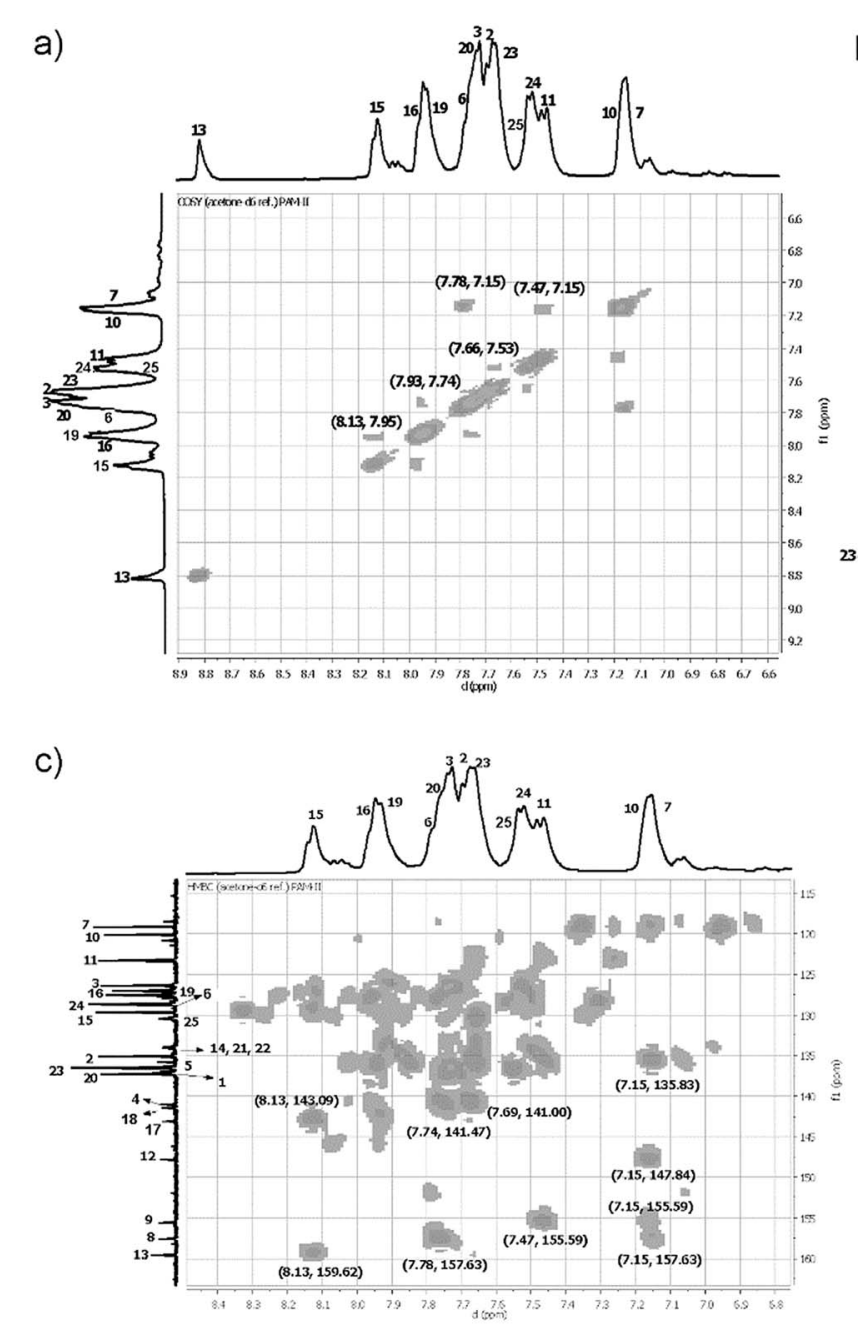

$\left(0.05 \mathrm{mg} \mathrm{mL}^{-1}\right)$ at $40{ }^{\circ} \mathrm{C}$ in order to obtain solutions. In all cases, clear solutions were obtained and then used in the GPC analysis.

Because no reports exist regarding the synthesis and characterization of poly(ether-azomethine)s containing $-\mathrm{PhSiR}_{2} \mathrm{Ph}-$ moieties in their chains ( $\mathrm{R}=$ methyl, phenyl), the solubility behavior of the new materials was compared with results obtained for non-silylated poly(ether-azomethine)s..34,35 These polymers have similar structural elements to the repetitive units of the prepared PAMs, including oxyether linkages and high aromatic content. Likewise, the $-\mathrm{PhSiR}_{2} \mathrm{Ph}-$ moieties are replaced by bulky and/or flexible central units promoted by the symmetric monomer moieties (Fig. 8).

In all cases, the authors report higher solubility in common organic solvents than those observed for the silylated PAMs. The PAMs-ref showed solubility even in THF and $\mathrm{CHCl}_{3}$ at room temperature. These differences can be mainly associated with the presence of oxyether bonds in the dialdehyde moiety, which favors the solubility process. Also, the biphenyl moieties of the silylated PAMs hinder the solubility of the chains. ${ }^{36}$ In this sense, the respective central elements of the silylated samples
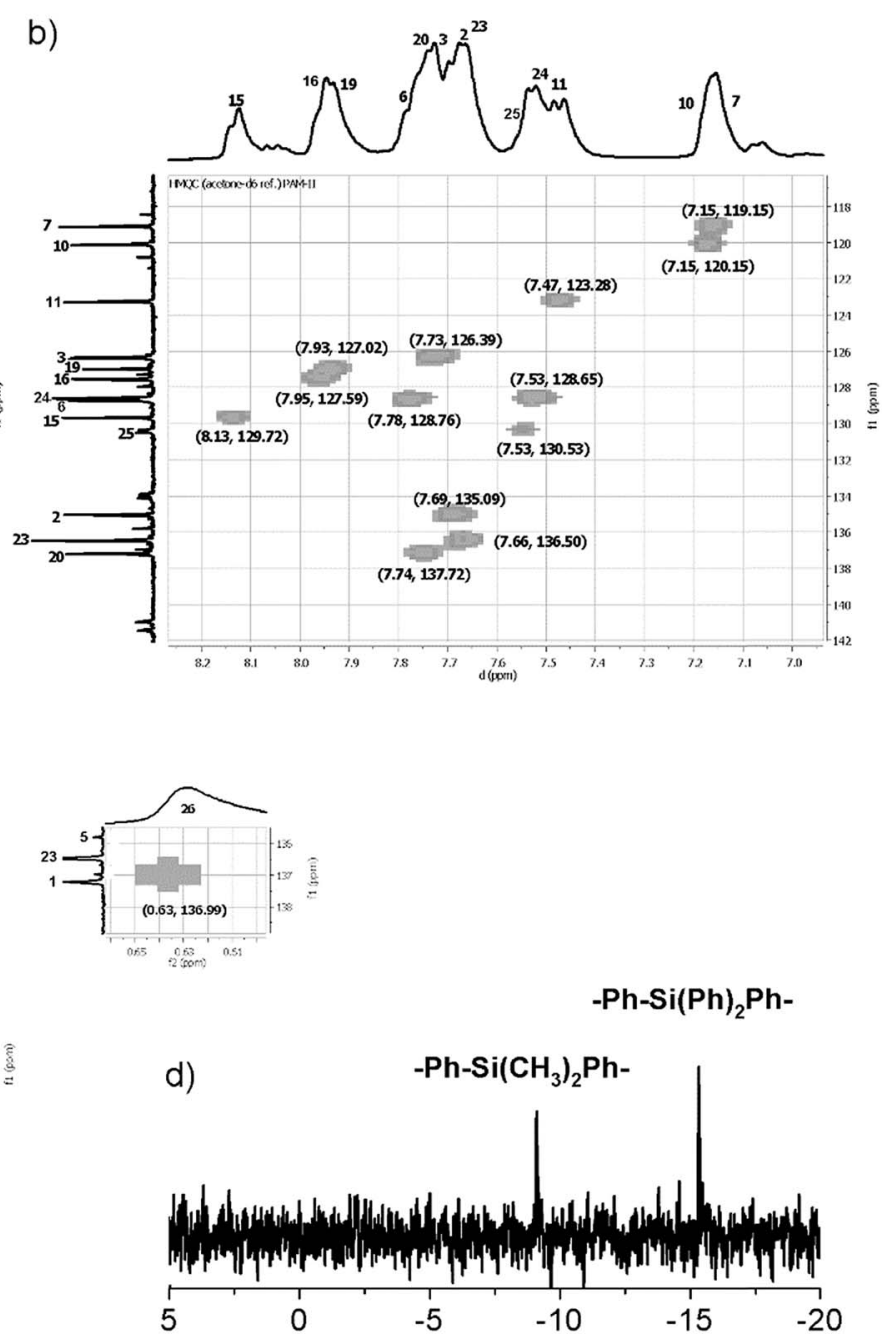

Fig. 7 NMR spectra (DMAc-acetone- $d_{6}$ ) of PAM-II. (a) COSY, (b) HMQC, (c) HMBC and (d) ${ }^{29}$ Si NMR spectra. 
Table 1 Solubility behavior of PAMs expressed by dissolution time and inherent viscosity ${ }^{a}$

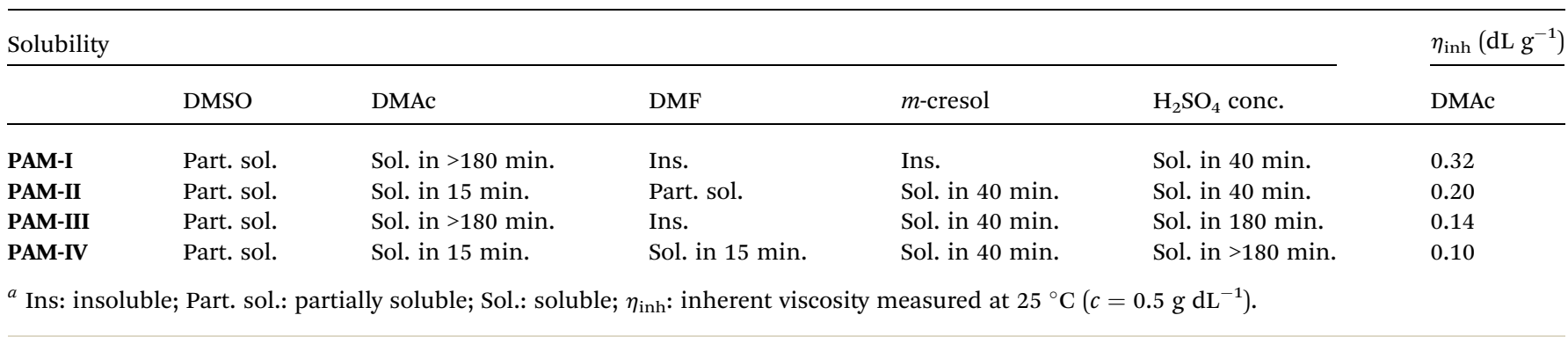

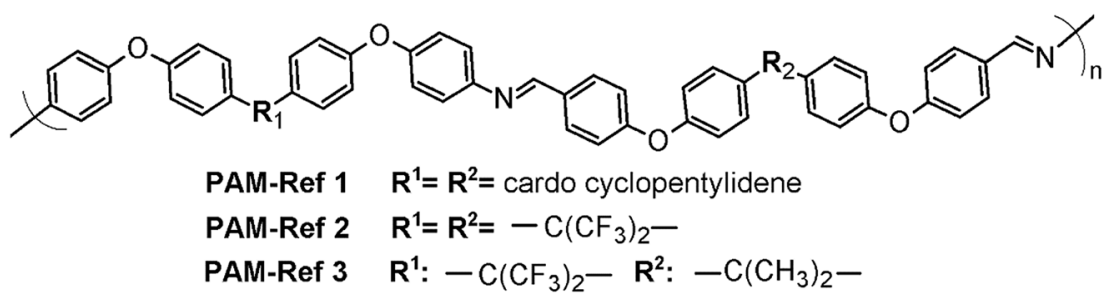

Fig. 8 General structure of the poly(ether-azomethine) used for comparison, emphasizing the natures of the central elements $\left(R^{1}\right.$ and $\left.R^{2}\right) .{ }^{34,35}$

and of the PAMs-ref may have similar effects on the studied properties because they are bulky elements that affect the packing of the polymeric chains.

Inherent viscosity measurements were used to establish an order of the size chain in the series. Although the samples showed solubility in concentrated sulfuric acid, this parameter was not determined in this medium because hydrolysis of the imine function was promoted by this reagent. According to a report by Morgan et al., ${ }^{7}$ although these measurements are made quickly, it is not possible to avoid hydrolysis of the chains, a process that is enhanced by the action of light.

Thus, the inherent viscosity was recorded at $25^{\circ} \mathrm{C}$ in DMAc $(c$ $\left.=0.5 \mathrm{~g} \mathrm{dL}^{-1}\right)$; values between 0.10 and $0.32 \mathrm{dL} \mathrm{g}^{-1}$ were obtained, which are characteristic of oligomers (Table 1). According to these data, PAM-I and PAM-II correspond to samples with larger sizes, while PAM-III and PAM-IV are smaller in size but are similar to each other. This observation is in accordance with the results of the FT-IR analysis because the AC parameter is related to the relative size of the chains; the smaller the AC value, the larger the chain. On the other hand, the GPC results obtained from DMF-LiBr solutions generally ratify these observations (Table 2). The obtained oligomers have 3 to 5 repetitive units, where PAM-I shows the highest molecular weight and PDI.

\section{Thermal analysis}

The thermal properties of the oligomers were evaluated by TGA and DSC techniques at $20{ }^{\circ} \mathrm{C} \mathrm{min}^{-1}$ in nitrogen atmosphere.

All the samples showed similar TG behaviors, with an initial decomposition temperature of around $450{ }^{\circ} \mathrm{C}\left(T_{\text {onset }}\right)$ (Fig. 9a). The $10 \%$ weight loss ( $\mathrm{TDT}_{10 \%}$ ) occured between $490{ }^{\circ} \mathrm{C}$ and $510{ }^{\circ} \mathrm{C}$. The char yields at $720{ }^{\circ} \mathrm{C}$ were similar in all samples, between $55 \%$ and $60 \%$; this is common for silylated samples,
Table 2 Molecular weights, degrees of polymerization and polydispersity indices of the oligomers measured by GPC

\begin{tabular}{lllll}
\hline & $M_{\mathrm{w}}\left(\mathrm{g} \mathrm{mol}^{-1}\right)$ & $M_{\mathrm{n}}\left(\mathrm{g} \mathrm{mol}^{-1}\right)$ & $\mathrm{DP}^{a}$ & $\mathrm{PDI}^{b}$ \\
\hline PAM-I & 9100 & 4800 & 5 & 1.90 \\
PAM-II & 6000 & 4200 & 4 & 1.42 \\
PAM-III & 4900 & 4000 & 4 & 1.23 \\
PAM-IV & 4900 & 3600 & 3 & 1.36
\end{tabular}

${ }^{a}$ Approximate degree of polymerization: $M_{\mathrm{n}} /$ molecular weight of repetitive unit. ${ }^{b}$ Polydispersity index: $M_{\mathrm{w}} / M_{\mathrm{n}}$.

principally due to the formation of non-volatile silicon oxides and non-oxidized organic material. ${ }^{30,37,38}$

Thermal stability studies performed on the poly(etherazomethines)s based on the decomposition point indicate that they are thermally stable. ${ }^{39}$ The high aromatic content due to the biphenyl moieties of both monomers allows inter-chain interactions that increase the thermal stability in a similar way to the effect discussed in the solubility section. When the lateral groups bonded to the silicon atoms were the same $\left(\mathrm{R}_{\mathbf{1}}=\right.$ $\mathrm{R}_{2}, \mathrm{CH}_{3}$ or phenyl group; PAM-I and PAM-IV), the samples were more stable than those with different groups $\left(\mathrm{R}_{1} \neq \mathrm{R}_{2}, \mathrm{CH}_{3}\right.$ or phenyl group; PAM-II and PAM-III). This behavior can be attributed to the structural symmetry of the repetitive units and the consequently higher packing forces between the chains. These results are summarized in Table 3.

The DSC curves (Fig. 9b) show transitions between $279{ }^{\circ} \mathrm{C}$ and $204{ }^{\circ} \mathrm{C}$, which are attributed to the glass transition temperature $\left(T_{\mathrm{g}}\right)$; however, for PAM-III, this thermal transition was not observed. No melting temperatures were observed in the analyses. In accordance with the results, the introduction of the flexible oxyether bond as a common structural element lowers the rigidity of the chains, resulting in low $T_{\mathrm{g}}$ values despite the high aromatic content of the repetitive units. 
a)

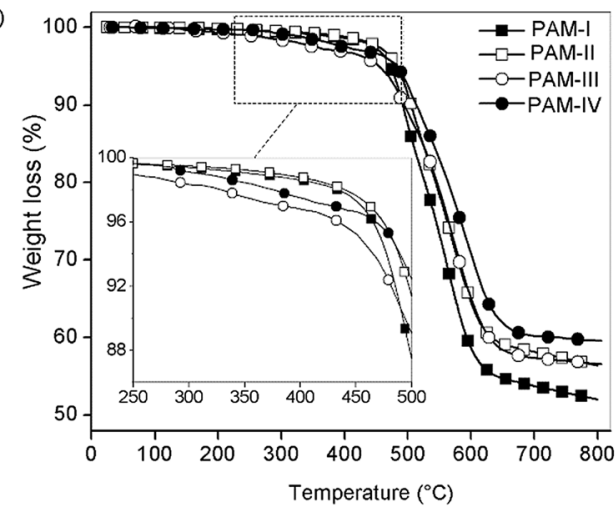

b)
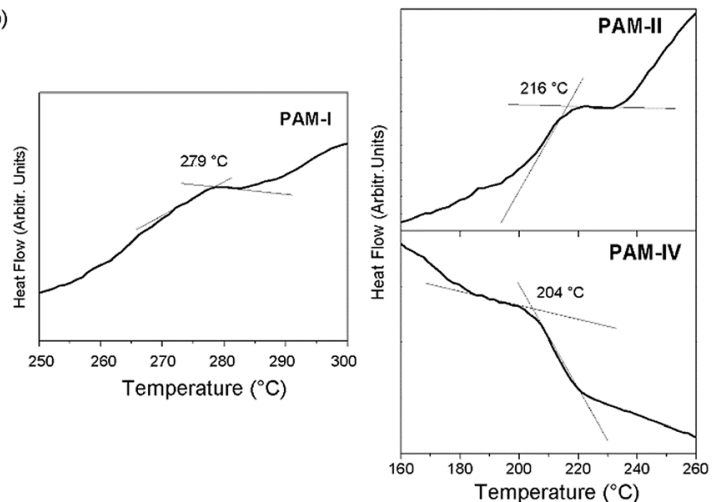

Fig. 9 Thermal studies: (a) TGA and (b) DSC of the samples.

Table 3 Thermal data obtained for the silylated-PAMs and PAMs-Ref ${ }^{a}$

\begin{tabular}{|c|c|c|c|c|}
\hline & \multirow{2}{*}{$\begin{array}{l}\text { DSC } \\
T_{\mathrm{g}_{\text {onset }}}\left({ }^{\circ} \mathrm{C}\right)\end{array}$} & \multicolumn{3}{|c|}{ Thermogravimetric analysis (TGA) } \\
\hline & & $\mathrm{TDT}_{10 \%}\left({ }^{\circ} \mathrm{C}\right)$ & $T_{\text {onset }}\left({ }^{\circ} \mathrm{C}\right)$ & $\begin{array}{l}\text { Charge yield } \\
\text { at } 720^{\circ} \mathrm{C}(\%)\end{array}$ \\
\hline PAM-I $\left(\mathrm{R}_{1}:-\mathrm{CH}_{3}, \mathrm{R}_{2}:-\mathrm{CH}_{3}\right)$ & 279 & 505 & 465 & 53 \\
\hline PAM-II $\left(\mathrm{R}_{1}:-\mathrm{CH}_{3}, \mathrm{R}_{2}:-\mathrm{Ph}\right)$ & 216 & 490 & 475 & 58 \\
\hline PAM-III $\left(\mathrm{R}_{1}:-\mathrm{Ph}, \mathrm{R}_{2}:-\mathrm{CH}_{3}\right)$ & n.o. & 495 & 455 & 57 \\
\hline PAM-Ref 2 & 211 & 481 & 420 & $0\left(\right.$ at $\left.630{ }^{\circ} \mathrm{C}\right)$ \\
\hline PAM-Ref 3 & 215 & 458 & 250 & $0\left(\right.$ at $\left.630{ }^{\circ} \mathrm{C}\right)$ \\
\hline
\end{tabular}

${ }^{a} T_{\text {onset: }}$ temperature at which polymer decomposition starts; n.o.: not observed.; n.r.: not reported.

Inside the series, the order of the $T_{\mathrm{g}}$ values correlates to the nature of the groups bonded to the silicon atoms and the sizes of the chains, which were established by GPC analysis. Thus, PAM-I, which contains only lateral methyl groups and has the highest average chain size, showed the highest $T_{\mathrm{g}}$ and therefore the greatest chain rigidity; meanwhile, PAM-IV, which contains four lateral phenyl groups and has the lowest average size, showed the lowest $T_{\mathrm{g}}$. In this sense, the smaller volume of a methyl group compared with a phenyl group promotes the interaction between chains, diminishing the internal rotation.

It is clear that the decomposition temperatures and the $T_{\mathrm{g}}$ values showed by the samples demonstrate an adequate thermal window in view of processing of this type of aromatic material.

If these thermal results are compared with those of nonsilylated-PAMs, similar tendencies in the $T_{\mathrm{g}}$ values can be seen in selected references ${ }^{\mathbf{3 4 3 5}}$ (Fig. 8); the bulkiest central element results in the lowest $T_{\mathrm{g}}$. On the other hand, if PAM-I is compared with PAM-Ref 1 or PAM-Ref 2, where the main difference is the atom of the central unit ( $\mathrm{Si} v s$. C, respectively), the silylated sample shows a higher $T_{\mathrm{g}}$. This result is contrary to the relationship of the volume of the atoms and can also be explained by the higher number of oxyether linkages in the reference samples, which promote higher flexibility of the chains. Regarding the $\mathrm{TDT}_{10 \%}$, the silylated samples show higher values, in accordance with the biphenyl moieties present in their repetitive units.

\section{UV/vis spectroscopy}

UV/vis spectroscopy was carried out in dilute DMSO solutions ( $c=0.5 \mathrm{mg} \mathrm{mL}^{-1}$ ) in the wavelength range between 270 and $440 \mathrm{~nm}$ (Fig. 10). The PAMs showed bands within 280 to $290 \mathrm{~nm}$ that can be assigned to the $\pi \rightarrow \pi^{*}$ transition of the aromatic rings of the main chain. On the other hand, a broad absorption can be found between $351 \mathrm{~nm}$ and $356 \mathrm{~nm}$, which was assigned to the $\mathrm{n} \rightarrow \pi$ transition of the imine groups. ${ }^{\mathbf{4 0 , 4 1}}$ The slight difference between these bands can be attributed to the modification of the chain planarity. That is, the conjugation is disrupted due to the presence of sterically hindered structures such as the phenyl rings as lateral groups in PAM-IV, showing bands centered at a lower wavelength compared with the other samples (structural variation of the molecule rigid backbone). These results are shown in Table 4.

From the UV-vis spectra, optical band gap values were obtained using eqn (1). ${ }^{42,43}$

$$
E_{\mathrm{g}}=1242 / \lambda_{\text {onset }}
$$

where $\lambda_{\text {onset }}$ is the onset wavelength, which was determined by the intersection of the two tangents on the absorption edges 
a)

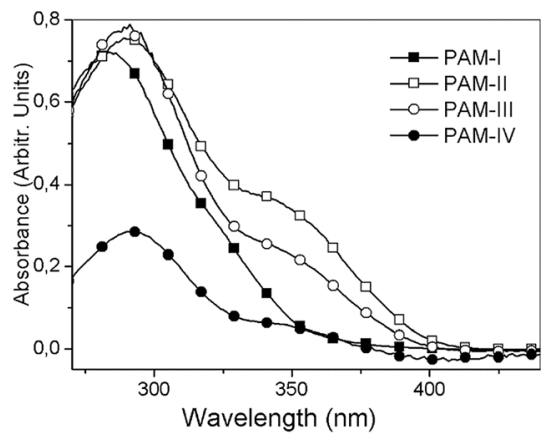

b)

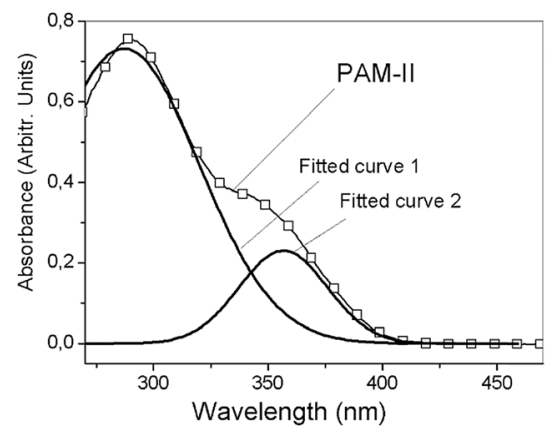

Fig. 10 (a) UV/vis spectra for PAM-I to PAM-IV in DMSO and (b) fitted curves for PAM-II.

Table 4 Bands and optical band gap values obtained from UV/vis DMSO solution spectra ${ }^{a}$

\begin{tabular}{lcccc}
\hline & & & & \\
& $1^{\circ}$ band & $2^{\circ}$ band & $(\mathrm{nm})$ & $E_{\mathrm{g}}(\mathrm{eV})$ \\
\hline PAM-I & 279.9 & - & 357.0 & 3.5 \\
PAM-II & 287.0 & 356.6 & 398.5 & 3.1 \\
PAM-III & 287.0 & 354.0 & 392.2 & 3.2 \\
PAM-IV & 290.6 & 351.7 & 377.5 & 3.3 \\
${ }^{a}$ Cutoff: $L_{\mathrm{O}}$ DMSO: $330 \mathrm{~nm}$ and $L_{1}: 285 \mathrm{~nm}$. & &
\end{tabular}

and was used to indicate the electronic transition of the onset wavelength. The calculated optical band gap values are shown in Table 4.

As can be observed, the optical band gaps are between 3.1 and $3.5 \mathrm{eV}$; these values are related to internal effects due to morphology and carrier transport, among other factors. ${ }^{44}$ PAM-I and PAM-IV showed the highest optical band gaps because the two groups bonded to their silicon atoms are identical $\left(R_{1}=R_{2}\right.$ $=$ methyl and phenyl, respectively). This structural symmetry around the silicon atom can strongly modulate the morphological effects on the measured parameter in a similar way to that proposed in the thermal section. On the other hand, small $E_{\mathrm{g}}$ values were obtained in the analysis of PAM-II and PAM-III. This behavior can be attributed, among other factors, to the reduction of steric hindrance and the greater delocalized $\pi$ electron density in the aromatic segments of the chains (conjugated polymers), which may involve electronic delocalization.

According to these results, it can be seen that the incorporation of phenyl groups can dramatically decrease the planarity of the system and therefore diminish the $\pi$-electron delocalization along the backbone.

\section{Atomic force microscopy and force spectroscopy}

PAM-I and PAM-II were chosen for the preparation of films using a spin-coating technique due to their high $M_{\mathrm{n}}$ values of 4800 and 4200, respectively. DMAc solutions of the samples were deposited over an activated silicon wafer substrate by spincoating with several cycles at a constant speed. Afterward, the films were subjected to vacuum to remove the remaining solvent.
Four experiments were performed by varing the rotational speed and time (speed in rpm/time in seconds): PAM-I (1) 2000/ 18 and 4000/60; PAM-II (2) 1000/18, 1500/18, 2000/18 and 4000/ 60, (3) 1500/18, 2000/18 and 4000/60, (4) 2000/18 and 4000/60.

In this preparation, the homogeneity of the layers is controlled by the physical structure of the sample. Additionally, the viscosity of the solvent and the rotational speed used for its deposition are factors that must be considered.

Fig. 11 shows AFM images of the PAM-I (1) and PAM-II (4) films. For PAM-I (1), the morphological structure presents a relatively homogeneous film formed by large clusters with shared boundaries, probably due to the high degree of solubility at the moment of material deposition. Also, it can be observed that the clusters are formed of small particles with similar sizes. On the other hand, PAM-II (4) shows a homogeneous surface, but with smaller clusters. Probably, the smaller size of the chains and the replacement of the methyl group by a phenyl group with greater volume do not promote the formation of higher structures.

During the spin coating process, solvent evaporation reduces the spin radius and increases the viscosity of the material; thus, it is impossible for centrifugal force to remove the remaining material from the wafer. Fig. 12a shows three different AFM images of PAM-II and their respective magnifications. The variation in the topography of the sample films is due to changes in the spinning speed during the deposition process. Fig. 12b shows the trends followed by the Young's modulus, the roughness, and the area increase percentage according to the initial spin speed used for the formation of each film. a)

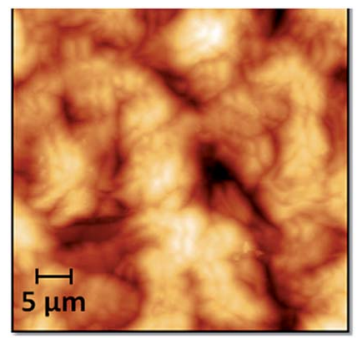

b)

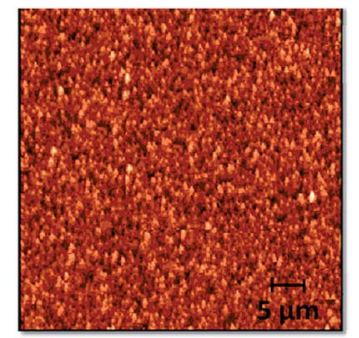

Fig. 11 AFM topographic images $\left(50 \times 50 \mu \mathrm{m}^{2}\right)$ of spin-coated films obtained from DMAc solutions: (a) PAM-I (1) and (b) PAM-II (4). 
At higher initial spinning speeds, the stiffness of the surface increases. This may be indirectly related to the roughness of the film. Higher spinning speeds produce homogeneous surfaces, decreasing the roughness but maintaining the particle size. The homogeneity of the films is also indicative of the presence of interconnected microstructures inside the material, producing network or boundary interactions that increase the Young's modulus of the surface. In the same way, by definition, the roughness increase produces an increase in the percentage of exposed surface area; both plots follow a similar tendency.

The four-point probe technique was used to measure the resistivities of the thin films. The setup consists of four point collinear probes, where a constant current is applied between the two outer probes and the voltage variation is measured in the inner probes. The surface resistivity was determined by eqn (2), where CF corresponds to a corrector factor based on the ratio of the probe and the substrate/film dimensions. ${ }^{45,46}$

$$
\sigma=\left(\frac{V}{I}\right) \times \mathrm{CF}
$$

The experiments were carried out using the following parameters: $I=6 \mathrm{~A} ; w$ (substrate thickness) $=0.0525 \mathrm{~cm}$, and $s$ (separation between the probes) $=0.1 \mathrm{~cm}$. Silicon wafer was used as a calibration standard and also as a substrate. The resistivity results obtained for the two samples are summarized in Table 5.

The refractive indices of PAM-I and PAM-II were 1.433 and 1.440, respectively; these were measured using an Abbe refractometer. These values are important for measuring the thicknesses of the films; using an ellipsometer, thicknesses near $500 \mathrm{~nm}$ were measured. These values were used to determine the resistivities of the films. The surface resistivities of the calibration standards (silicon wafers) are certified by the company (Siegert Wafer GmbH).

Table 5 Electrical studies using the four-point probe technique

\begin{tabular}{lccc}
\hline & $\begin{array}{l}\text { Voltage } \\
(\mathrm{V})\end{array}$ & $\begin{array}{l}\text { Resistivity } \\
(\Omega \mathrm{cm})\end{array}$ & $\begin{array}{l}\text { Average }(\Omega \\
\mathrm{cm})\end{array}$ \\
\hline PAM-I (1) & 3.56 & 2.19 & 2.19 \\
PAM-II (2) & 32.24 & 19.85 & 20.45 \\
PAM-II (3) & 35.84 & 22.06 & \\
PAM-II (4) & 31.59 & 19.45 & 10.02 \\
Silicon wafer_1 (bare) & 15.32 & 9.43 & \\
Silicon wafer_2 (bare) & 17.24 & 10.61 &
\end{tabular}

a)

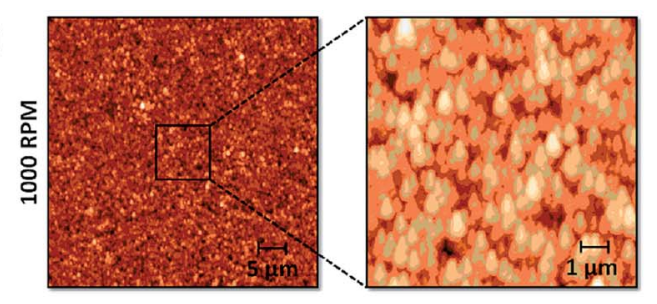

PAM-II (2)

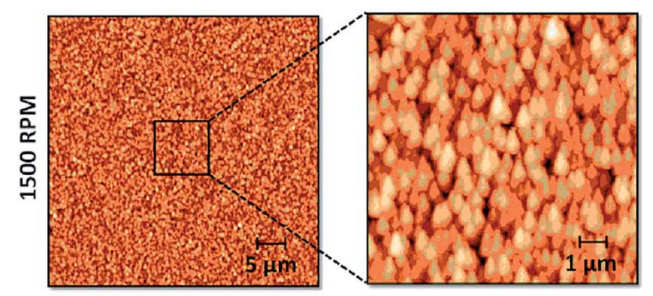

PAM-II (3)

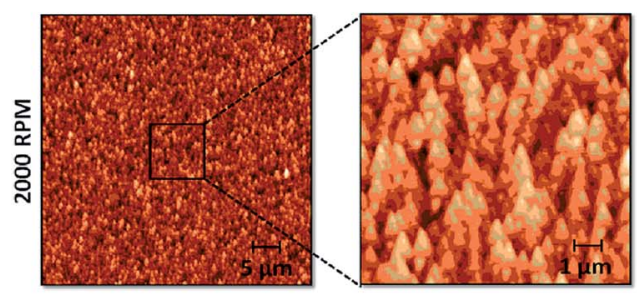

PAM-II (4)

b)

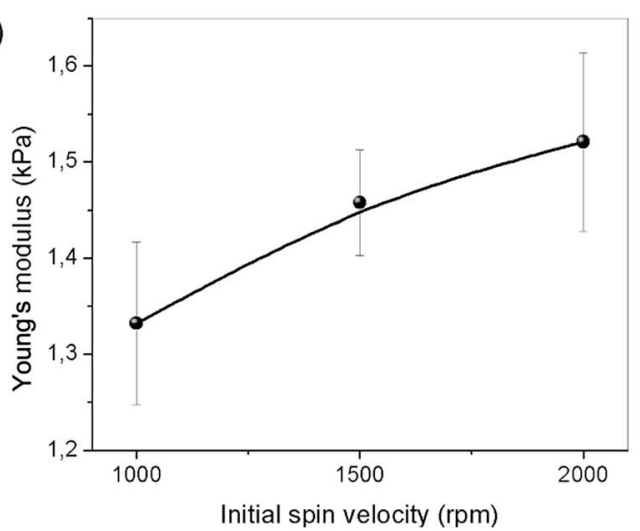

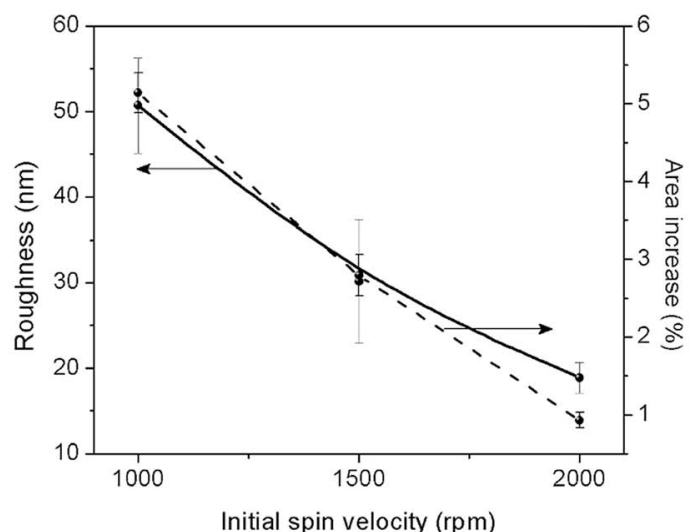

Fig. 12 (a) AFM topographic images $\left(50 \times 50 \mu \mathrm{m}^{2}\right.$ and $\left.10 \times 10 \mu \mathrm{m}^{2}\right)$ of spin-coated PAM-II films obtained from DMAc solutions and (b) Young's modulus, roughness, and area increase percentage obtained from the analysis of the images. 
Scientific reports have shown that the resistivity of a specimen increases with decreasing thickness. ${ }^{47}$ However, in this case, the thickness of the sample was not strongly affected by the rate of deposition, probably due to the high viscosity of the polymer-low volatile solvent (DMAc) solution. Table 5 shows the results obtained for PAM-I and PAM-II. The first sample has low resistivity $(2.19 \Omega \mathrm{cm})$, while PAM-II showed a resistivity close to $20 \Omega \mathrm{cm}$. As can be seen, these results are not related to the rotation speed used for their deposition. Also, these results are in disagreement with those obtained from the UV-vis analysis (Table 4), mainly due to intrinsic differences of the samples under study. That is, a sample deposited over a silicon wafer as a thin film presents structural differences that are reflected in its morphology at the surface level; the sample in solution, observed by UV/vis spectroscopy, does not possess this property (Fig. 10).

\section{Conclusions}

From four bifunctional monomers, two diamines and two dialdehydes containing diphenylsilane and biphenyl moieties, four oligomeric poly(ether-azomethine)s were synthesized and structurally characterized using NMR and FT-IR spectroscopy. The FT-IR spectroscopy results suggested that the qualitative relationship between the sizes of the chains of these samples agreed with their inherent viscosity values and GPC results, which established that PAM-I is the sample with the highest molecular weight. The solubility results showed an effect of the nature of the organic groups bonded to the silicon atoms, which modify the packing forces between the chains. In this sense, the replacement of the lateral methyl groups by phenyl groups with greater volume enhanced the solubilities of the samples. Optical properties measured by UV-vis (optical absorption) showed band gap values between 3.1 and $3.5 \mathrm{eV}$, which are insufficient for potential applications in the optoelectronics field. Additionally, the thermal analysis showed that all the samples were highly stable, with $\mathrm{TDT}_{10 \%}>490{ }^{\circ} \mathrm{C}$ and an adequate thermal window of processability between TDT and $T_{\mathrm{g}}$.

Two samples were used to generate films from DMAc solutions using a spin-coating technique. Thus, morphological and resistivity studies were performed using AFM and the fourprobe method, respectively. The speed of rotation did not affect the thickness of the films; therefore, no significant changes in their conductivities were observed. However, the surface morphology of the films was affected by the structure of the oligomeric poly(ether-azomethine)s and also by the spin speed used for deposition; an increase in the stiffness of the surface was observed with increasing initial spin speed.

\section{Conflicts of interest}

There are no conflicts to declare.

\section{Acknowledgements}

The authors acknowledge financial assistance from Fondo Nacional de Desarrollo Científico y Tecnológico, FONDECYT, through Project 1150157, and FONDEQUIP, through Project EQM120021. P. A. Sobarzo thanks CONICYT for fellowship No. 21151317.

\section{References}

1 M. L. Petrus, R. K. Bouwer, U. Lafont, D. H. K. Murthy, R. J. Kist, M. L. Böhm, Y. Oliver, T. J. Savenije, L. D. A. Siebbeles, N. C. Greenham and T. J. Dingemans, Polym. Chem., 2013, 4, 4182-4191.

2 M. Grigoras and C. O. Catanescu, J. Macromol. Sci., Polym. Rev., 2004, 44, 131-173.

3 A. Iwan and D. Sek, Prog. Polym. Sci., 2008, 33, 289-345.

4 G. F. Dálelio, J. V. Crivello, R. K. Schoenig and T. F. Huemmer, J. Macromol. Sci., Part A, 1967, 1, 1161-1249.

5 C.-J. Yang and S. A. Jenekhe, Macromolecules, 1995, 28, 11801196.

6 R. H. Friend, R. W. Gymer, A. B. Holmes, J. H. Burroughes, R. N. Marks, C. Taliani, D. D. C. Bradley, D. A. Dos Santos, J. L. Bredas, M. Logdlund and W. R. Salaneck, Nature, 1999, 397, 121-128.

7 P. W. Morgan, S. L. Kwolek and T. C. Pletcher, Macromolecules, 1987, 20, 729-739.

8 M. Bruma and B. Schulz, J. Macromol. Sci., Part C: Polym. Rev., 2006, 41, 1-40.

9 K. S. Yook and J. Y. Lee, Adv. Mater., 2012, 24, 3169-3190.

10 Q. Zhao, W. Zhang, Z. Fan, J. Li, X. Chen, G. Luo and X. Zhang, Synth. Met., 2015, 204, 70-75.

11 F. Xu, J. H. Kim, H. U. Kim, J. H. Jang, K. S. Yook, J. Y. Lee and D. H. Hwang, Macromolecules, 2014, 47, 7397-7406.

12 D. Sun, X. Zhou, H. Li, X. Sun, Y. Zheng, Z. Ren, D. Ma, M. R. Bryce and S. Yan, J. Mater. Chem. C, 2014, 2, 8277-8284.

13 H. C. Yeh, C. H. Chien, P. I. Shih, M. C. Yuan and C. F. Shu, Macromolecules, 2008, 41, 3801-3807.

14 P. Cui, X. F. Jing, Y. Yuan and G. S. Zhu, Chin. Chem. Lett., 2016, 27, 1479-1484.

15 P. Puthiaraj and W. S. Ahn, Ind. Eng. Chem. Res., 2016, 55, 7917-7923.

16 J. Moncada, C. A. Terraza, L. H. Tagle, D. Coll, P. Ortiz, G. Pérez, J. G. de la Campa, C. Alvarez and A. TundidorCamba, Eur. Polym. J., 2017, 91, 354-367.

17 Y. Liu, J. Y. L. Lai, S. Chen, Y. Li, K. Jiang, J. Zhao, Z. Li, H. Hu, T. Ma, H. Lin, J. Liu, J. Zhang, F. Huang, D. Yu and H. Yan, J. Mater. Chem. A, 2015, 3, 13632-13636.

18 J. Yang, W. He, K. Denman, Y.-B. Jiang and Y. Qin, J. Mater. Chem. A, 2015, 3, 2108-2119.

19 L. Li, H. Ren, Y. Yuan, G. Yu and G. Zhu, J. Mater. Chem. A, 2014, 2, 11091-11098.

20 B. Zhang, P. Zhang, H. Zhang, C. Yan, Z. Zheng, B. Wu and Y. Yu, Macromol. Rapid Commun., 2017, 38, 1700110.

21 I. F. MacSim, E. N. Dragoi, M. Cazacu and S. Curteanu, J. Appl. Polym. Sci., 2015, 132, 42552.

22 M. F. Zaltariov, M. Cazacu, C. Racles, V. Musteata, A. Vlad and A. Airinei, J. Appl. Polym. Sci., 2015, 132, 41631.

23 A. Airinei, M. Homocianu, A. Vlad and M. Cazacu, J. Mol. Liq., 2013, 186, 171-178. 
24 C. Racles, M. Silion, A. Arvinte, M. Iacob and M. Cazacu, Des. Monomers Polym., 2013, 16, 425-435.

25 M. Palewicz, A. Iwan, M. Sibinski, A. Sikora and B. Mazurek, Energy Procedia, 2011, 3, 84-91.

26 M.-F. Zaltariov, M. Cazacu, S. Shova, C.-D. Varganici, L. Vacareanu, V. Musteata and A. Airinei, Des. Monomers Polym., 2014, 17, 668-683.

27 J. J. Roa, G. Oncins, J. Díaz, F. Sanz and M. Segarra, Recent Pat. Nanotechnol., 2011, 5, 27-36.

28 P. Klapetek, M. Valtr, D. Neças, O. Salyk and P. Dzik, Nanoscale Res. Lett., 2011, 6, 514.

29 I. M. Tidswell, B. M. Ocko, P. S. Pershan, S. R. Wasserman, G. M. Whitesides and J. D. Axe, Phys. Rev. B, 1990, 41, 1111-1128.

30 G. W. Gale, R. J. Small and K. A. Reinhardt, Aqueous Cleaning Surface Conditioning Processes, in Handbook on Silicon Wafer Cleaning Technology, William Andrew Inc., USA, 2nd edn, 2008.

31 C. A. Terraza, L. H. Tagle, F. Concha and L. Poblete, Des. Monomers Polym., 2007, 10, 253-261.

32 C. A. Terraza, L. H. Tagle, A. Tundidor-Camba, C. M. González-Henríquez, D. Coll and M. A. Sarabia, RSC Adv., 2015, 5, 28515-28526.

33 C. A. Terraza, L. H. Tagle, C. Contador, A. Tundidor-Camba and C. M. González-Henríquez, Polym. Bull., 2014, 71, 1101-1115.
34 S. S. Ankushrao, V. N. Kadam, V. P. Ubale and A. A. Ghanwat, International Journal of Engineering and Science Invention, 2016, 5, 67-73.

35 P. K. Gutch, S. Banerjee, D. C. Gupta and D. K. Jaiswall, J. Polym. Sci., Part A: Polym. Chem., 2001, 39, 383-388.

36 S. Banerjee, P. K. Gutch and C. Saxena, Des. Monomers Polym., 1999, 2, 135-142.

37 C. A. Terraza, L. H. Tagle, A. Tundidor-Camba, C. M. González-Henríquez, M. A. Sarabia-Vallejos and D. Coll, RSC Adv., 2016, 6, 49335-49347.

38 C. A. Terraza, L. H. Tagle, D. Muñoz, A. Tundidor-Camba, P. A. Ortiz, D. Coll, C. M. González-Henríquez and I. A. Jessop, Polym. Bull., 2016, 73, 1103-1117.

39 I. Kaya, A. Avci and K. Temizkan, Korean J. Chem. Eng., 2015, 31, 777-786.

40 D. Şenol and K. İsmet, J. Saudi Chem. Soc., 2017, 21, 505-516. 41 D. Dilek, F. Doğan, A. Bilici and İ. Kaya, Thermochim. Acta, 2011, 518, 72-81.

42 M. M. Ahmida and S. H. Eichhorn, ECS Trans., 2010, 25, 110.

43 T. Daimon and E. Nihei, Materials, 2013, 6, 1704-1717.

44 H. Cha, C. E. Park, S. K. Kwon and T. K. An, Org. Electron., 2017, 45, 263-272.

45 F. M. Smits, Measurements of Sheet Resistivity with the Four-Point Probe, Bell Syst. Tech. J., 1958, 37, 711-718.

46 L. B. Valdes, Proc. IRE, 1954, 420-427.

47 D. B. Tanner and D. C. Larson, Phys. Rev., 1968, 3, 652-655. 